\title{
Optimal pricing for electricity retailers based on data-driven consumers' price-response
}

\author{
Román Pérez-Santalla ${ }^{1} \cdot$ Miguel Carrión $^{2} \cdot$ Carlos Ruiz $^{3}$ (D)
}

Received: 1 October 2021 / Accepted: 5 February 2022 / Published online: 22 February 2022

(c) The Author(s) 2022

\begin{abstract}
In the present work, we tackle the problem of finding the optimal price tariff to be set by a risk-averse electric retailer participating in the pool and whose customers are price sensitive. We assume that the retailer has access to a sufficiently large smart-meter dataset from which it can statistically characterize the relationship between the tariff price and the demand load of its clients. Three different models are analyzed to predict the aggregated load as a function of the electricity prices and other parameters, as humidity or temperature. More specifically, we train linear regression (predictive) models to forecast the resulting demand load as a function of the retail price. Then, we will insert this model in a quadratic optimization problem which evaluates the optimal price to be offered. This optimization problem accounts for different sources of uncertainty including consumer's response, pool prices and renewable source availability, and relies on a stochastic and risk-averse formulation. In particular, one important contribution of this work is to base the scenario generation and reduction procedure on the statistical properties of the resulting predictive model. This allows us to properly quantify (data-driven) not only the expected value but the level of uncertainty associated with the main problem parameters. Moreover, we consider both standard forward-based contracts and the recently introduced power purchase agreement contracts as risk-hedging tools for the retailer. The results are promising as profits are found for the retailer with highly competitive prices and some possible improvements are shown if richer datasets could be available in the future. A realistic case study and multiple sensitivity analyses have been performed to characterize the risk-aversion behavior of the retailer considering price-sensitive consumers. It has been assumed that the energy procurement of the retailer can be satisfied from the pool and different types of contracts. The obtained results reveal that the risk-aversion degree of the retailer strongly influences contracting decisions, whereas the price sensitiveness of consumers has a higher impact on the selling price offered.
\end{abstract}

Keywords Electricity retailer · Price-sensitive consumers · Risk aversion · Smart meter data $\cdot$ Stochastic programming $\cdot$ Time-of-use rate

Extended author information available on the last page of the article 
Mathematics Subject Classification 90-11 · 91B74 · 90C15

\section{Introduction, state of the art and contributions}

Intermittent and renewable units are the generation technologies that are being most installed nowadays in power systems (IRENA 2021). The capital costs of these units are moderately high, but they have the advantage of not burning fuel in the electricity production process, which results in low operation costs. As a consequence of this, electricity prices in the spot markets can be very low if the total power output of the renewable units is high. On the contrary, electricity prices can be elevated if the renewable production is low. These high prices can be even enlarged if extra charges are required to be paid by fossil-fuel-based generators to penalize the emission of greenhouse gases. For instance, this is the case of generating power plants in Europe that are forced to participate in the EU Emissions Trading System (European 2021), which prices have doubled during year 2021 reaching the 64.37 euro/ton on September 27 th. As a result, the volatility of electricity prices has increased significantly during the last years in those power systems with high intermittent production, (Dong et al. 2019).

The effect of the volatility of electricity prices may be particularly negative for the economic objectives of electricity retailers. In a power system framework, retailers are entities that purchase electricity in different markets and through bilateral contracts to sell it afterwards to the end users of electricity. The financial revenue obtained by retailers comes from the sale of electricity to their clients, whereas the costs incurred by retailers are those derived from its procurement of electricity. Then, if the purchase price in the electricity markets is higher than expected, the acquisition cost of electricity for retailers will be also high and they may even suffer from losses. In the same manner, this situation may also happen if the selling price offered by retailers to their clients is too low. In this case, the revenue obtained from selling energy could be smaller than the procurement cost of the retailer. Moreover, with the progressive integration of smart grid technologies within distribution systems, we may expect a increasing price-demand elasticity of consumers, which needs to be accurately characterized. As a consequence of this, the appropriate determination of the electricity procurement strategy and the selling price offered to their clients by retailers is key for achieving profitability.

In this work, we investigate the decisions (energy procurement and selling price determination strategies) to be taken by a retailer to maximize its expected profit within a time of use (ToU) tariff. A ToU rate is that one which the selling price offered to clients vary for each trading period of the day. The idea behind the ToU tariff is to communicate to clients the price offered so that they can choose to use more or less electricity within the time period that the offer is valid. We set this investigation in the British electricity market (OFGEM 2021). For that we use real world smart-meters consumption dataset which spans almost the whole year of 2013 (London 2013). The dataset is comprised of half-hourly electric demand and price data for 1000 British households, along with some 
other related variables such as apparent temperature, humidity or precipitation. Once the response of consumers to electricity prices is characterized and modeled through a predictive model, a risk-averse two-stage stochastic programming model is formulated to determine the energy procurement and selling price by retailers. Different energy procurement sources are considered in this problem.

As more countries are installing smart meters to record household consumption (Akhavan-Hejazi and Mohsenian-Rad 2018) retailers have now more information than ever to cater prices to consumers. Some previous works have approached this price design by clustering customers with similar behaviours to offer a similar tariff (Yang et al. 2018). Another approach taken by Yang et al. (2013) is to formulate a game between the retailer and different types of consumers (such as industrial and households). Reference (Feng et al. 2020) models the retailer-consumer interaction as Stackelberg competition (retailer as leader and customers as followers) to produce a bilevel optimization problem.

Many works have been also developed to formulate the different problems faced by electricity retailers under uncertainty. Some of the most relevant models derived in the last years are described below. In this sense, reference (Carrión et al. 2007) formulated for the first time a two-stage stochastic programming problem to decide the purchasing strategy and the selling price offered by an electricity retailer in a medium-term planning horizon. This work was expanded in Carrión et al. (2007), where a bilevel problem was formulated to take into account explicitly the decision-making problem faced by electricity end users that desire to select optimally their electricity suppliers. Additionally, reference (Hatami et al. 2011) incorporated time-of-use rates in the problem formulated in Carrión et al. (2007) modelling also the elasticity of the demand. The authors of Kettunen et al. (2010) developed a novel multi-stage stochastic optimization approach to decide the contract portfolio of retailers. The numerical results obtained using this procedure indicate that the correlation between price and load has a direct influence on the decisions of the retailer. In Reference (García-Bertrand 2013), it is proposed a novel procedure that considers selling price modifications to incentive load shifts intended to increase the expected profit of the retailer. It is observed that this new price scheme is able to increase the profit of retailers, while the payments of the consumers are reduced. The authors of Nojavan et al. (2017) formulate the bilateral contracting and selling price determination of a retailer in a smart grid framework considering distributed generation, energy storage systems and a demand response program. In the same manner that (García-Bertrand 2013), this work concludes that the demand response program is able to increase the expected profit of the retailer and decrease the selling price offered to the clients. In Reference (Deng et al. 2020), a procedure for designing real-time pricing rates is proposed for different types of consumers. The novelty of this procedure is the usage of the downside risk constraints method to decide the risk strategy of the retailer. The numerical results indicate that the risk faced by the retailer may be efficiently controlled using this method. Finally, the recent work (Feng et al. 2020) derives a data-driven approach to design time-of-use tariffs by modeling a Stackelberg game between the retailer and strategic consumers. The obtained tariffs are able to shave demand peaks and fill demand valleys. 
What we propose in this work is to first identify the sensitivity of customers using historical data to model customer's responses to prices (exhibited by the demand). Customer elasticity has become increasingly important as it can help in smoothing the peaks of consumption which have become ever increasing as electric demand grows and renewable sources play a bigger role in the system. Furthermore, new technologies on the electric grid have allowed to make this real-time communication viable (US Energy department 2022). To measure the sensitivity of customers to the price offered we will take the coefficient of a linear regression model corresponding to the price variable. Several models of different size and with different variables are analyzed to figure out which variables better improve the prediction accuracy. Among the most meaningful variables were lagged values of the electric demand and temperature data (Hyndman and Athanasopoulos 2018). After selecting 3 main models of different size (1 with 8 predictors, another with 30 and finally, a complex model which used a different linear model for each time period to be predicted Fan and Hyndman 2012) comparisons are made to find the most suitable for the present work. These comparisons focused not only in the accuracy of the model but also on the impact within the model of the price predictor. Moreover, we seek to characterize statistically the distributions of the estimated model parameters. This information will be latter used to generate a representative set scenarios for the stochastic programming problem.

The next step is to formulate the profit maximization problem faced by retailers in the British electricity market. Like most European markets, the British electricity market is mainly based on the interactions through different trading floors between producers (energy generating plants of various types) who sell the energy to large consumers or to retailers, who sell the energy to the final consumers (individuals or companies who purchase electric energy for consumption). In this work, we have established three market mechanisms for the retailer to purchase energy. First, the retailer may purchase energy from the pool, which is a market place where producers, retailers and consumers trade electricity with a typical planning horizon of 1 day divided in hourly or half-hourly periods. We consider the pool to be an unlimited source of energy (there is no cap to the amount of energy that can be purchased from the pool) which is a fair approximation as most producers in Britain participate in this market. The second way in which the retailer may purchase energy is through a forward base contract which is a bilateral agreement between a producer and a retailer where a certain amount of energy is purchased during a certain time frame for the agreed price. In this type of contract, the producer must deliver the amount of energy contracted, so it is normally linked to non-renewable sources where the power producer can always deliver the promised energy (dispatchable units). Finally, the third option we have modeled for the retailer to purchase energy from is a power purchase agreement (PPA) which is a different type of bilateral contract where the power delivered in each trading period is not necessarily fixed, as it can depend on other factors, such as weather for renewable sources (non-dispatchable) (Conejo et al. 2010). An example of PPA may be a bilateral contract signed with a solar photovoltaic power plant, which production is different in each hour of the day. Considering these three trading floors, a risk-averse two-stage stochastic programming problem is formulated to decide the energy procured by the retailer in each available 
trading floor and to determine the selling price offered to their clients. The stochastic variables considered in this model are: (1) pool prices, (2) energy availability of the PPA and (3) response of end users of electricity to the prices offered by the retailer. The most important decisions to obtain using this formulation are the power contracted from forward and PPA contracts and the selling prices offered by the retailer to their clients. Considering a ToU rate, the selling prices offered by the retailer can be different for each half-hour period and they are indexed to pool prices. The riskaversion of the retailer is modeled using the conditional value-at-risk (CVaR) of the profit distribution.

The main contributions of this work are fivefold:

(1) to use real smart meter data to quantify the price-load response of consumers in terms of predictive linear regression model.

(2) to use the statistical properties of above model and real world datasets to generate a meaningful set of scenarios of the uncertain parameters: price sensitivity of consumers, renewable availability and pool prices.

(3) to consider both forward-based contracts (expensive but certain availability) with the recently introduced PPA contracts (cheap but uncertain availability), as risk-hedging tools for a retailer participating in a pool market.

(4) to explicitly insert the forecasting model in (1) as a constraint in the two-stage stochastic problem faced by the retailer together with the scenarios derived in (2).

(5) to study through realistic simulations the main properties and interactions between PPA and forward-based contract prices, and the impact of level of consumers elasticity on the retailer's optimal tariffs and profits.

We believe that the data-driven methodology presented in this work is general and can be applied to other price-load smart-meter datasets. We hope that these will become more and more available in the near future with the progressive integration of smart-grid technologies.

\section{Price-demand data-driven characterization}

As mentioned before, the first step in solving the optimization problem is to characterize, as accurately as possible, the demand-response level of price-sensitive consumers, and how this is impacted by other exogenous variables.

We propose a novel data-driven approach to (i) infer the relationship between the consumer's demand and the retail price (tariff) and (ii) insert it explicitly in the retailer's decision-making model (stochastic optimization).

Let us assume that we have a sample (data set) of $n$ observations of the type $S_{N}=\left\{\left(y^{1}, x_{1}^{1}, \ldots, y_{k}^{1}\right), \ldots,\left(y^{n}, x_{1}^{n}, \ldots, y_{k}^{n}\right)\right\}$, where $y, x_{j}^{i} \in \mathbb{R}, i=1, \ldots, n, j=1, \ldots, k$. Lets also assume that both the response variable $y$ (demand) and the first of the explanatory variables, $x_{1}$ (price), are decision variables within our stochastic model, 
while the remaining variables $x_{-1}=\left\{x_{2}, \ldots, x_{k}\right\}$ can be considered as contextual information (covariates), known at the time of decision making. Hence, we seek to characterize the relationship between them through a prediction model of the type $y=f\left(x_{1} \mid x_{-1}\right)$ that can be inserted within the retailers optimization problem. In particular, we can consider the following linear model:

$$
y=\beta_{0}+\beta_{1} x_{1}+\sum_{j=2}^{k} \beta_{j} x_{j}
$$

where $\beta_{0}, \beta_{1}, \ldots, \beta_{k}$ are the linear regression coefficients to be estimated from the sample $S_{N}$ by Ordinary Least Squares (OLS) or any other statistical approach. Once estimated, the resulting model can be expressed as

$$
y=\hat{\beta}_{1} x_{1}+\hat{D}\left(x_{-1}\right)
$$

where $\hat{D}\left(x_{-1}\right)=\hat{\beta}_{0}+\sum_{j=2}^{k} \hat{\beta}_{j} x_{j}+\epsilon$ is fully characterized by the realization of the contextual information $x_{-1}$, being $\epsilon$ the residual (error). If $y$ and $x_{1}$ are considered optimization variables, the data-driven function (1) can be easily incorporated in the optimization problem in the form of a linear constraint, and without increasing substantially the computational complexity of the resulting optimization problem.

Moreover, considering a linear model like (1) within an stochastic optimisation framework, presents two important advantages:

(i) the statistical distribution of the residuals $\epsilon$ and the estimated $\hat{\beta}_{j}$ for $j=0, \ldots, k$ is well characterized, so that realistic data-driven scenarios of this linear relationship can be generated.

(ii) $\hat{\beta}_{1}$ can be directly interpreted as the degree by which demand $(y)$ is altered by a marginal change of the tariff $\left(x_{1}\right)$, which is closely linked to the concept of demand elasticity with important economic implications.

In the present model, the contextual information would include meteorological and calendar information, together with past realizations of prices and demands (lags). Similarly, in the stochastic optimization contest, we will assume that the retail price $x_{1}$ and the demand-response by the consumers $y$ are second stage decisions and hence, conditioned by the realization of uncertainty (scenario dependent). Moreover, model (1) will be extended with a temporal dependency, as we employ a half-hourly time resolution. This will be further explained in the following sections.

\subsection{Scenario generation}

For the stochastic optimization problem, we need the most accurate characterization of its uncertain parameters. In the current work, we will consider that these include half hourly pool prices, solar availability, and the price coefficient $\left(\beta_{1}\right)$ of the linear model introduced in the previous section. We deal with the task of creating this representation by utilizing a large number of randomly generated scenarios. Each of these is generated by concatenating random possible realizations of pool prices, 
solar availability and the price coefficient of the linear model. The process for generating the pool and solar scenarios is dealt with in Appendix 1. To generate scenarios for the regression coefficient of the price predictor, we utilize the distribution of the parameter estimate $\hat{\beta}_{1}$ instead of its expected value $\mathbb{E}\left[\hat{\beta}_{1}\right]$. For clarity, we make use of the matrix formulation of the linear system (1) as follows:

$$
\mathbf{y}=\mathbf{X} \boldsymbol{\beta}+\boldsymbol{\epsilon}, \quad \text { where } \quad \mathbf{y}, \boldsymbol{\epsilon} \in \mathbb{R}^{n \times 1}, \quad \mathbf{X} \in \mathbb{R}^{n \times k}, \quad \boldsymbol{\beta} \in \mathbb{R}^{k \times 1}
$$

The first step is to identify the distribution of $\hat{\beta}_{1}$ under OLS and the assumption that the model residuals are i.i.d. with $\mathbb{E}\left[\epsilon_{i}\right]=\mu_{\epsilon}, \mathbb{V} \operatorname{ar}\left[\epsilon_{i}\right]=\sigma_{\epsilon}^{2}$ for $i=1, \ldots, n$. Using the Hajek-Sidak CLT and assuming that its condition is satisfied (a heuristic argument for this assumption can be found in Appendix 1 for the data set employed in Sect. 4), the limit distribution for $\hat{\beta}_{1}$ is Normal:

$$
\hat{\beta}_{1} \rightarrow \mathcal{N}\left(\beta_{1}+\mu_{\epsilon} \sum_{j=1}^{n}\left[\left(\mathbf{X}^{T} \mathbf{X}\right)^{-1} \mathbf{X}^{T}\right]_{1 j}, \sigma_{\epsilon}^{2} \sum_{j=1}^{n}\left[\left(\mathbf{X}^{T} \mathbf{X}\right)^{-1} \mathbf{X}^{T}\right]_{1 j}^{2}\right)
$$

Replacing $\beta_{1}$ with its estimate $\mathbb{E}\left[\hat{\beta}_{1}\right]$ and $\mu_{\epsilon}$ with the residual sample mean $\overline{\boldsymbol{\epsilon}}$ which can be assumed to be 0 , the distribution can be written as:

$$
\hat{\beta}_{1} \rightarrow \mathcal{N}\left(\mathbb{E}\left[\hat{\beta}_{1}\right], \sigma_{\epsilon}^{2} \sum_{j=1}^{n}\left[\left(\mathbf{X}^{T} \mathbf{X}\right)^{-1} \mathbf{X}^{T}\right]_{1 j}^{2}\right)
$$

Scenarios for the regression coefficient of the price predictor are then sampled from this distribution.

\section{Stochastic optimization problem}

This section describes the mathematical formulation of the retailer problem. This problem is formulated using a risk-averse two-stage stochastic approach in which the demand-response of price-sensitive consumers is explicitly characterized. The final problem is recast as a quadratic programming problem.

\subsection{Notation}

The notation used to formulate the optimization problem is included below for quick reference.

\subsection{Indices and sets}

- $\quad D$ : Set of days, indexed by $d$ 
- $T$ : Set of half-hourly periods, indexed by $t$

- $T_{d}$ : Set of time periods for day $d$

- $\Omega$ : Set of scenarios, indexed by $\omega$

\subsection{Variables}

- $c_{t, \omega}$ : Retailer's cost for each half-hourly period and each scenario

- $d_{t, \omega}$ : Total demand of consumers contracting power from the retailer per halfhourly period and scenario

- $\quad p^{\mathrm{B}}$ : Energy contracted by the retailer from forward base contracts (fixed quantity per half-hourly period $p^{\mathrm{B}}$ with a fixed price $\bar{\lambda}^{\mathrm{B}}$ )

- $\quad p^{\mathrm{C}, \mathrm{PPA}}$ : Energy contracted by the retailer from the PPA contract for each halghourly period

- $\quad p_{t, \omega}^{\mathrm{P}}$ : Energy bought by the retailer from the pool per half-hourly period and scenario at price $\bar{\lambda}_{t, \omega}^{\mathrm{P}}$

- $p_{t, \omega}^{\text {PPA }}$ : Energy purchased from PPA contract with a renewable power producer subject to the energy contracted $p^{\mathrm{C}, \mathrm{PPA}}$. The energy purchased from this contract at each half-hourly period and scenario depends on the availability of the renewable source, $A_{t, \omega}^{\mathrm{PPA}} \in[0,1]$. The energy purchased through the PPA contract is priced at $\bar{\lambda}^{\mathrm{PPA}}$

- $r_{t, \omega}$ : Retailer's revenue for each half-hourly period and each scenario

- $s_{\omega}$ : Auxiliary variables for CVaR formulation

- $\eta$ : Auxiliary variable for CVaR formulation equivalent to the VaR at the optimal solution of the stochastic problem.

- $\lambda_{t}^{\mathrm{E}}$ : Variable part of the price offered by the retailer

- $\lambda_{t, \omega}^{\mathrm{R}}$ : Price offered per half-hourly period and scenario to consumers by the retailer

- $\Theta$ : Set of all the above decision variables

\subsection{Parameters}

- $A_{t, \omega}^{\mathrm{PPA}}$ : Availability of the renewable source for each half-hourly period and each scenario

- $\hat{D}_{t}$ : Half-hourly demand effects outside the price-coefficient interaction

- $\pi_{\omega}$ : Probability assigned to each scenario

- $P_{\max }^{\mathrm{B}}$ : Maximum energy available for purchase from the forward base contracts in each half-hourly period

- $P_{\max }^{\text {PPA }}$ : Maximum energy available for purchase from the PPA contract in each half-hourly period

- $\alpha$ : Fraction of the distribution to be used in the CVaR calculation (as $1-\alpha)$

- $\beta_{\omega}$ : Coefficient of the price predictor for each scenario

- $\gamma$ : Parameter for establishing bounds for every element of the variable part of the price $\left(\lambda_{t}^{\mathrm{E}}\right)$

- $\bar{\lambda}^{\mathrm{E}}$ : Mean value of the variable part of the price $\left(\lambda_{t}^{\mathrm{E}}\right)$

- $\bar{\lambda}^{\mathrm{B}}$ : Price for energy contracted from forward base contracts 
- $\quad \lambda_{t \omega}^{\mathrm{P}}$ : Half-hourly pool prices for each scenario

- $\quad \bar{\lambda}^{\mathrm{PPA}}$ : Price for energy contracted from the PPA contract

- $\chi$ : Parameter weighing the importance of the CVaR against the profit

\subsection{Formulation}

The formulation for the decision-making problem faced by a retailer that desires to determine its energy procurement strategy and the selling prices offered to clients is included below. This formulation considers explicitly a data-driven model to characterize and anticipate the price sensitiveness of the retailer's clients. The electricity procurement options of the retailer are purchasing from (i) the pool at variable and uncertain price, (ii) a forward contract with fixed and identical prices and quantities for every half-hourly period, and (iii) a PPA with a renewable energy provider that offers a variable and uncertain production at fixed price.

As stated above, the formulation of this problem corresponds to a risk-averse two-stage stochastic programming approach. The uncertain parameters considered in this problem are the pool prices, $\lambda_{t, \omega}^{\mathrm{P}}$, the elasticity of the demand of the clients of the retailer, $\beta_{\omega}$, and the availability of the PPA, $A_{t, \omega}^{\mathrm{PPA}}$. These parameters are characterized using a set of scenarios $\omega \in \Omega$. The risk-aversion of the retailer is modeled by the CVaR of the profit. The first-stage decisions of this problem are the retailerdependent part of the selling price, $\lambda_{t}^{\mathrm{E}}$, and the half-hourly energy contracted from forward and PPA contracts, $p^{\mathrm{B}}$ and $p^{\mathrm{C}, \mathrm{PPA}}$, respectively.

$$
\begin{gathered}
\max _{\Theta}(1-\chi) \sum_{\omega \in \Omega} \sum_{t \in T} \pi_{\omega}\left(r_{t, \omega}-c_{t, \omega}\right)+\chi\left(\eta-\frac{1}{1-\alpha} \sum_{\omega \in \Omega} \pi_{\omega} s_{\omega}\right) \\
\text { s.t. } \quad r_{t, \omega}=\lambda_{t, \omega}^{\mathrm{R}} d_{t, \omega}, \quad \forall t, \omega \\
c_{t, \omega}=\bar{\lambda}_{t, \omega}^{\mathrm{P}} p_{t, \omega}^{\mathrm{P}}+\bar{\lambda}^{\mathrm{B}} p^{\mathrm{B}}+\bar{\lambda}^{\mathrm{PPA}} p_{t, \omega}^{\mathrm{PPA}}, \quad \forall t, \omega \\
d_{t, \omega}=\beta_{\omega} \lambda_{t, \omega}^{\mathrm{R}}+\hat{D}_{t}, \quad \forall t, \omega \\
d_{t, \omega}=p_{t, \omega}^{\mathrm{P}}+p^{\mathrm{B}}+p_{t, \omega}^{\mathrm{PPA}}, \quad \forall t, \omega \\
0 \leq p^{\mathrm{B}} \leq P_{\text {max }}^{\mathrm{B}} \\
p_{t, \omega}^{\mathrm{PPA}}=A_{t, \omega}^{\mathrm{PPA}} p^{\mathrm{C}, \mathrm{PPA}}, \quad \forall t, \omega \\
0 \leq p^{\mathrm{C}, \mathrm{PPA}} \leq P_{\max }^{\mathrm{PPA}}
\end{gathered}
$$




$$
\begin{gathered}
\lambda_{t, \omega}^{\mathrm{R}}=\lambda_{t}^{\mathrm{E}}+\left(\lambda_{t, \omega}^{\mathrm{P}}-\bar{\lambda}^{\mathrm{E}}\right), \quad \forall t, \omega \\
\frac{1}{\left|T_{d}\right|} \sum_{t \in T_{d}} \lambda_{t}^{\mathrm{E}}=\bar{\lambda}^{\mathrm{E}} \\
(1-\gamma) \bar{\lambda}^{\mathrm{E}} \leq \lambda_{t}^{\mathrm{E}} \leq(1+\gamma) \bar{\lambda}^{\mathrm{E}}, \quad \forall t \\
\eta-\sum_{t \in T}\left(r_{t, \omega}-c_{t, \omega}\right) \leq s_{\omega}, \quad \forall \omega \\
0 \leq s_{\omega}, \quad \forall \omega
\end{gathered}
$$

where $\Theta=\left\{d_{t, \omega}, p^{\mathrm{B}}, p^{\mathrm{C}, \mathrm{PPA}}, p_{t, \omega}^{\mathrm{P}}, p_{t, \omega}^{\mathrm{PPA}}, r_{t, \omega}, s_{\omega}, \lambda_{t}^{\mathrm{E}}, \lambda_{t, \omega}^{\mathrm{R}}, \eta\right\}$ is the set of optimization variables.

The objective function (5a) is the weighted sum of the expected profit of the retailer and the $\mathrm{CVaR}$ of the profit. The CVaR is used to model the risk faced by the retailer and it is equal to the average value of the $(1-\alpha)$ scenarios with lowest profit. Parameter $\chi \in[0,1]$ is used to model the risk aversion of the retailer. If $\chi=0$, the $\mathrm{CVaR}$ term is neglected and the retailer behaves as a risk-neutral decision maker. Values of $\chi$ greater than 0 represent different risk-aversion degrees. The expected profit is computed as the sum of the profits over the set of scenarios multiplied by their respective probabilities, $\pi_{\omega}$. Note that we make use of the linear CVaR formulation proposed by Rockafellar and Uryasev $(2000,2002)$.

Equation (5b) computes the revenue $r_{t, \omega}$ obtained by the retailer for each time period $t$ and scenario $\omega$ as the demand $d_{t, \omega}$ multiplied by the price of the corresponding time period $\lambda_{t, \omega}^{\mathrm{R}}$. The cost incurred by the retailer in each period $t$ and scenario $\omega, c_{t, \omega}$, is formulated in Eq. (5c) and it is equal the sum of the cost of purchasing energy in the pool, $\bar{\lambda}_{t, \omega}^{\mathrm{P}} p_{t, \omega}^{\mathrm{P}}$, the cost of purchasing through the bilateral contract, $\bar{\lambda}^{\mathrm{B}} p^{\mathrm{B}}$, and the cost of purchasing from the PPA, $\bar{\lambda}^{\mathrm{PPA}} p_{t, \omega}^{\mathrm{PPA}}$.

Equation (5d) is a scenario-dependent extension of the linear model introduced in (1), and represents that the total demand of the consumers is given by the linear relationship between the price offered by the retailer, $\lambda_{t, \omega}^{\mathrm{R}}$, and the consumer response quantified using the coefficient of the price predictor, $\beta_{\omega}$, plus the demand that is independent of the selling price, $\hat{D}_{t}$, which is characterized by contextual information.

The energy balance of the retailer in each period and scenario is formulated by Eq. (5d), which enforces that the total demand of the clients of the retailer in each period and scenario has to be equal to the energy purchased in the pool plus the energy contracted through forward and PPA contracts. Constraints (5f) limit the energy contracted from the forward contract. Constraints (5g) and (5h) formulate the energy contracted through the PPA. Note that the energy associated with period $t$ and scenario $\omega, p_{t, \omega}^{\mathrm{PPA}}$, depends on the energy contracted $p^{\mathrm{PPA}}$ and on the availability of the production $A_{t, \omega}^{\mathrm{PPA}}$. Note that the availability $A_{t, \omega}^{\mathrm{PPA}}$ ranges in 
the interval $[0,1]$. This parameter is used to model availability of the renewable source for a given period and scenario. Therefore, if $A_{t, \omega}^{\mathrm{PPA}}=1$, the whole capacity contracted will be available for the retailer. On the contrary, if $A_{t, \omega}^{\mathrm{PPA}}=0$, it is not possible to obtain energy from the PPA in period $t$ and scenario $\omega$.

Constraints (5i)-(5k) formulate mathematically the ToU rate offered by the retailer to its clients. As it is usual in many European countries, this paper assumes that the selling prices associated with the ToU rate are indexed to pool prices. Then, as stated by constraints (5i), the price that the clients of the retailer have to pay in each half-hourly period, $\lambda_{t, \omega}^{\mathrm{R}}$ is equal to a price term decided by the retailer, $\lambda_{t}^{\mathrm{E}}$, plus an additional term dependent on the pool price, $\left(\lambda_{t, \omega}^{\mathrm{P}}-\bar{\lambda}^{\mathrm{E}}\right)$. Symbol $\bar{\lambda}^{\mathrm{E}}$ refers to the average value of the price term decided by the retailer, which is a known parameter of the problem (5j). Additionally, parameter $\bar{\lambda}^{\mathrm{E}}$ is also used to bound the value of the price term $\lambda_{t}^{\mathrm{E}}$ in each half-hourly period by constraints (5k). Parameter $\gamma$ in (5k) is used to establish these limits. Because of pool prices are characterized as a stochastic parameter modeled using a set of scenarios, note that the selling price $\lambda_{t, \omega}^{\mathrm{R}}$ is also dependent of the scenario index $\omega$.

Finally, constraints $(51)$ and $(5 \mathrm{~m})$ are used to model the CVaR (Rockafellar and Uryasev 2000, 2002). The value of $\eta$ equals the Value at Risk (VaR) at the optimal solution of problem (5).

Observe that problem $(5 \mathrm{a})-(5 \mathrm{~m})$ is a quadratic linear programming problem that can be solved using commercial solvers with global optimality guarantees. The quadratic term results from replacing the demand $d_{t, \omega}$ expressed in (5d) into constraint (5b), which results in $r_{t, \omega}=\beta_{\omega}\left(\lambda_{t, \omega}^{\mathrm{R}}\right)^{2}+\hat{D}_{t} \lambda_{t, \omega}^{\mathrm{R}}$.

\section{Case study}

\subsection{The dataset}

The dataset utilized was collected by the UK Power Networks for investigating the differences between grid users with a dynamic tariff and those with a standard tariff. The half-hourly data (London 2013) taken from homes in London in 2013, has around 1100 customers using a dynamic tariff (referred to as ToU, standing for Time of Use, from now on) and the remainder of the dataset, around 4500 customers, using a standard tariff. We are currently interested only in those with the ToU tariff as for those we can include the price predictor. The information about the electric price for a certain time period was given to customers through the use of smart meters which would offer three different prices depending on external factors. The prices issued are High (67.20 p/kWh), Low (3.99 p/kWh) and normal (11.76 p/ $\mathrm{kWh}$ ), where $\mathrm{p}$ stands for pence or $1 / 100$ of a British pound (£). The dataset includes half-hourly information about electric consumption for the ToU customers and multiple other variables to be used as predictors, including price. This is not the most adequate setting to infer the price-response of customers i.e., it will be convenient to observe a higher number of different price levels or even a continuous price signal. However, we show through Sect. 4.2 that the resulting inferred price-response 


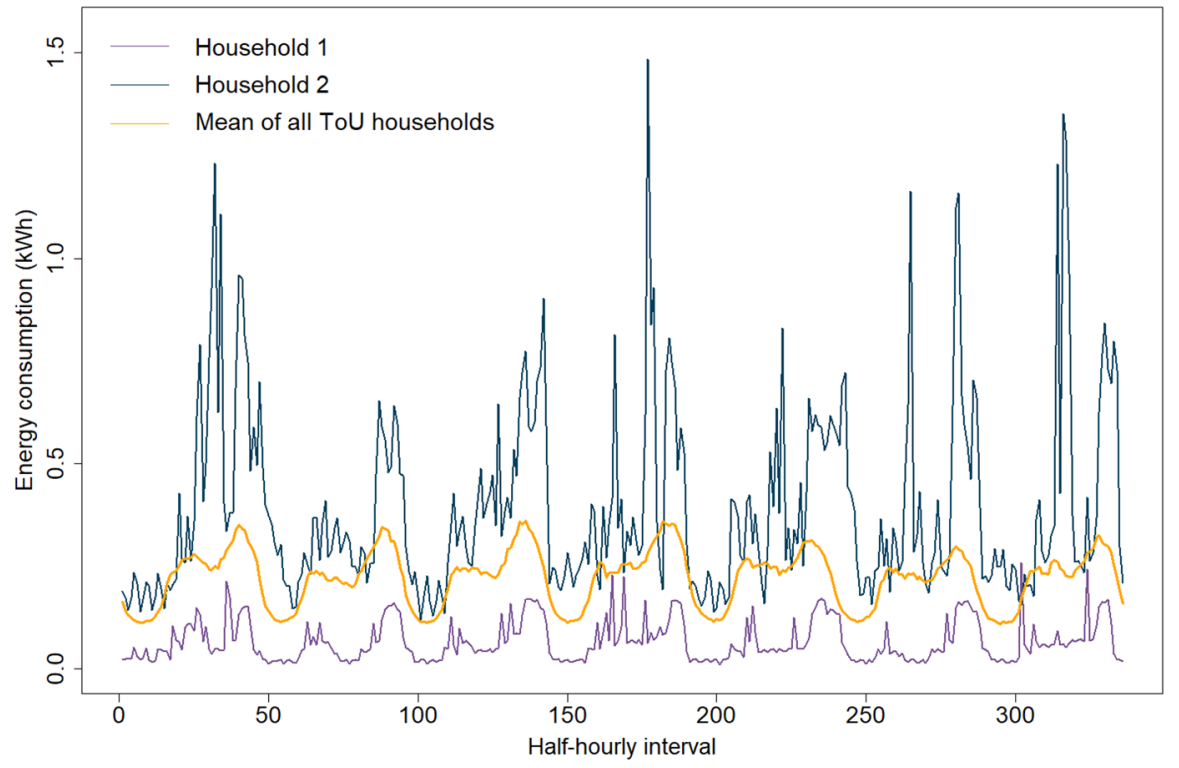

Fig. 1 Plot of energy consumption of two random households and mean of consumption of households with ToU tariff for the week starting the first of April of 2013

is low but still statistically meaningful for some models. In other words, we are able to characterize a significant change in the expected load when customers are offered a different price level for the next hour (e.g., from the Low to the High retail price). This flexibility may arise from several factors: the possibility of displacing the use of some appliances to cheaper hours (e.g., washing machine), to avoid electricity waste in expensive hours (e.g., turn off the lights that are not needed), the potential use of electrical storage, etc.

Figure 1 depicts two households' energy consumption during a week. Comparing these 2 profiles with the mean consumption of the 1100 consumers (also represented in the same figure), it is clear that individual household consumption signals present more noise and variability than said mean. This makes characterizing and predicting the mean consumption pattern much more feasible than those of individual households.

This is one of the most complete openly available datasets where variable realtime electricity prices are offered to disaggregated consumers. However, there are a few shortcomings which must be addressed. The dataset spans only 1 year (2013) and in the present work, we are interested in considering the retailer's options for a month, this month being chosen arbitrarily as December. This means that we are left with only 11 months of data to fit the model. If we focus on linear models, predicting accurately the half hourly demand for an entire month is challenging, as variables such as lags of the electric consumption of the customers or weather data are necessary. For this reason we have reached the compromise of developing a model which can predict accurately the demand for the next $24 \mathrm{~h}$ (that is, the nearest lagged variables are at least 48 half-hourly periods away from the period to be predicted). 
What this means is that the predictions would require information which would be unknown at the time of the decision. A remedy could be found using variables from different years (however, the dataset does not allow for this as it is limited to 1 year) or iterate through the testing window so that predictions for a given day can be used as input data for the following one. As solving the optimization problem for just 1 day, which would comply with the model's predictors, was deemed too unrealistic, we have chosen to operate on the assumption that this shortcoming could be fixed with a richer dataset. This assumption is well justified as retailers would rapidly accumulate real time (and price dependent) consumption data as smart meters penetrate among its consumers. Hence, we have proceeded to work with December as our testing month where the different contracts and tariffs signed in the first stage hold.

There is also a problem stemming from the price being offered in just 3 levels, with the normal price being the most prevalent by a large margin. This necessarily makes the price predictor have low impact with respect to the others if any reasonable degree of accuracy in predictions is desired. The importance of this predictor will be analysed for the models explored, but it is certain that with a dataset with more different price signals, as explained above, the impact of the predictor would be of greater significance.

For these reasons, in the following sections, we want to provide a general methodology that can be employed by electricity retailers with access to historical consumption data from its price-sensitive costumers. Hence, the aim is not to obtain the best forecasting model, but rather to explore how this data-driven model can be included implicitly on the decision-making process of a retailer and better characterize (statistically) potential uncertainty sources.

\subsection{Predictive models: demand vs price}

We explore three different linear models to predict the aggregate consumers demand as a function of the electricity price and other covariates, for the month of December. These are dubbed Small Model, Large Model and Combined Model. A brief description of each model follows.

\subsubsection{Small model}

This is the simplest model. The predictors used are the following:

- Apparent temperature: The apparent temperature of the half-hourly period. It is a combination of air temperature, wind speed and humidity.

- Humidity: The atmospheric humidity of the half-hourly period.

- Demand lags: Two lags are used, the electric demand of 48 periods earlier (1 day) and the electric demand of 336 periods earlier (1 week).

- Month: The month to which the half-hourly period belongs. 
- Price: The price signal communicated to the customers for the half-hourly period. Intentionally kept as a continuous variable despite its categorical nature to further utilize its corresponding coefficient.

Also, all three interactions between Month, Demand lags and Apparent temperature are included.

\subsubsection{Large model}

This is a larger model (30 predictors) expanding on the previous Small Model with the following additional predictors:

- Temperature: The temperature of the half-hourly period.

- Splines: A fit of a 6 knot cubic spline to the data to quantify annual effects in demand. The corresponding curve to the test data is a continuation of this fitted curve. This approach stems from Hyndman and Fan (2010).

- Day of week: 6 dummy variables coding the day of the week for the half-hourly period.

- Demand lags: Lagged electric demand in intervals of 48, 96, 144, 192, 240, 288 and 336 half-hourly periods to the corresponding period.

- Temperature lags: Lagged temperature in intervals of 48, 96, 144, 192, 240, 288 and 336 half-hourly periods to the corresponding period.

- Minimum demand: Minimum electric demand registered in the previous $24 \mathrm{~h}$ to the half-hourly time period.

- Maximum demand: Maximum electric demand registered in the previous $24 \mathrm{~h}$ to the half-hourly time period.

- Mean demand: Mean electric demand registered in the previous $24 \mathrm{~h}$ to the halfhourly time period.

- Minimum temperature: Minimum temperature registered in the previous $24 \mathrm{~h}$ to the half-hourly time period.

- Maximum temperature: Maximum temperature registered in the previous $24 \mathrm{~h}$ to the half-hourly time period.

- Mean temperature: Mean temperature registered in the previous $24 \mathrm{~h}$ to the halfhourly time period.

- Holiday: Dummy variable representing if the half-hourly period corresponds to a date categorized as a holiday.

\subsubsection{Combined model}

This model utilizes the same predictors as the Large Model. However, this model is actually a combination of 48 different linear models each being tasked with the prediction of a single half-hourly period of the day, giving it added flexibility. This model was inspired by the work of Hyndman and Fan (2010) and Fan and Hyndman (2012). 
Table 1 Table representing the aforementioned metrics of model performance

\begin{tabular}{llllll}
\hline & MAE train & RMSE train & MAE test & RMSE test & $R^{2}$ \\
\hline Small model & 12.38 & 18.92 & 14.54 & 20.80 & 0.945 \\
Large model & 12.42 & 18.18 & 13.51 & 19.53 & 0.949 \\
Combined model & 8.99 & 12.86 & 13.22 & 19.18 & 0.974 \\
\hline
\end{tabular}

\subsubsection{Model comparisons}

We start by comparing the metrics of Root Mean Squared Error (RMSE), Mean Squared Error (MAE), and $R^{2}$ of model performance across the three models.

In Table 1, we can see that although the Combined Model's overall performance is better, there appear to be some symptoms of over fitting, as the differences between test and train metrics are much larger than in both other models. Barring this possible over fitting problem in the Combined Model, the models' performances are reasonably good across the board. Given this similar performance, we now concern ourselves with the impact of the price predictor for each model. Since we are trying to model price sensitivity in customers, a larger impact of the price predictor is desired. To quantify this impact, the following analysis are made.

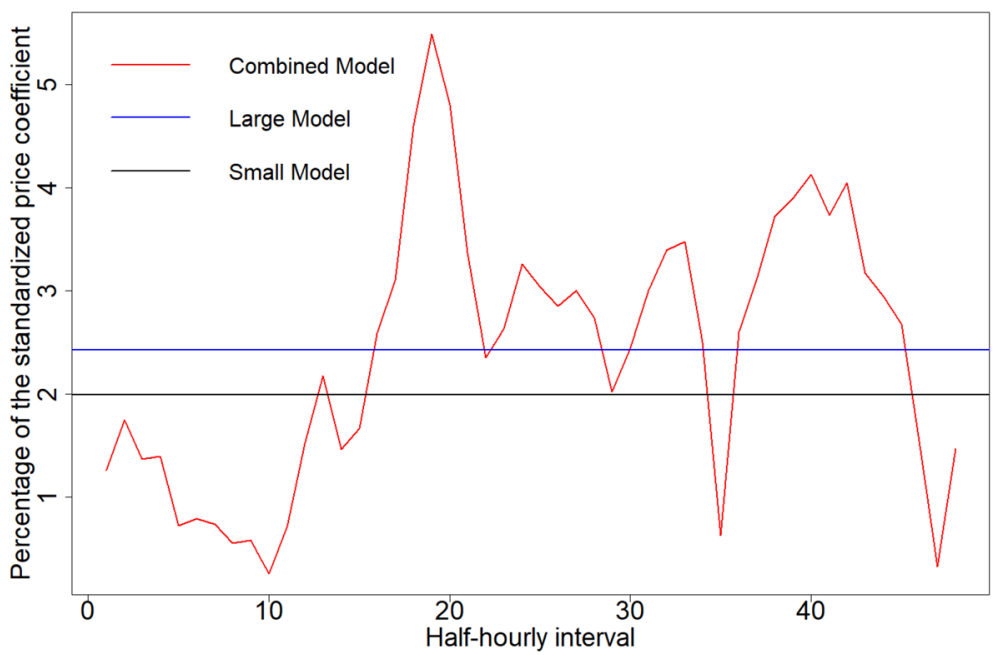

Fig. 2 Plot of the relative percentage of the standardized price coefficient with respect to the rest for each model 


\subsubsection{Impact of the standardized coefficient}

We first analyze the impact of the standardized coefficient for the price predictor (for clarity denoted as $\beta_{\text {price }}$ ) with respect to the rest of standardized predictors. That is $\left|\beta_{\text {price }}\right| / \sum_{j=1}^{k}\left|\beta_{j}\right| \times 100$.

It is worth noting that since the Combined Model is composed of 48 linear models, the representation of the aforementioned model is composed of 48 standardized coefficients. Figure 2 shows the comparison between the models.

It is clear from the figure that the impact of the price predictor through this metric is low for all three models. It is interesting to compare the Large and Small models and note that even though the Large Model has around three times as many predictors, the percentage taken up by the standardized price predictor is larger than that of the Small Model. Upon further inspection, this is due to some of the lag predictors taking much higher values than in the Large Model.

\subsubsection{Changes in RMSE and MAE when dropping the price predictor}

To further explore the impact of the price predictor, we now investigate the impact in the models' performances when the price predictor is removed and the models are fitted again. We compare the differences in RMSE and MAE for the models with and without the price predictor across both the train and test sets. Once again, the Combined Model's differences are represented for each half-hourly interval for the train set, however, for the test set, the number of data points was deemed insufficient to represent differences for each half-hourly period, so this differences are averaged across all the linear models which compose the aforementioned model. The results are plotted in Figs. 3 and 4.

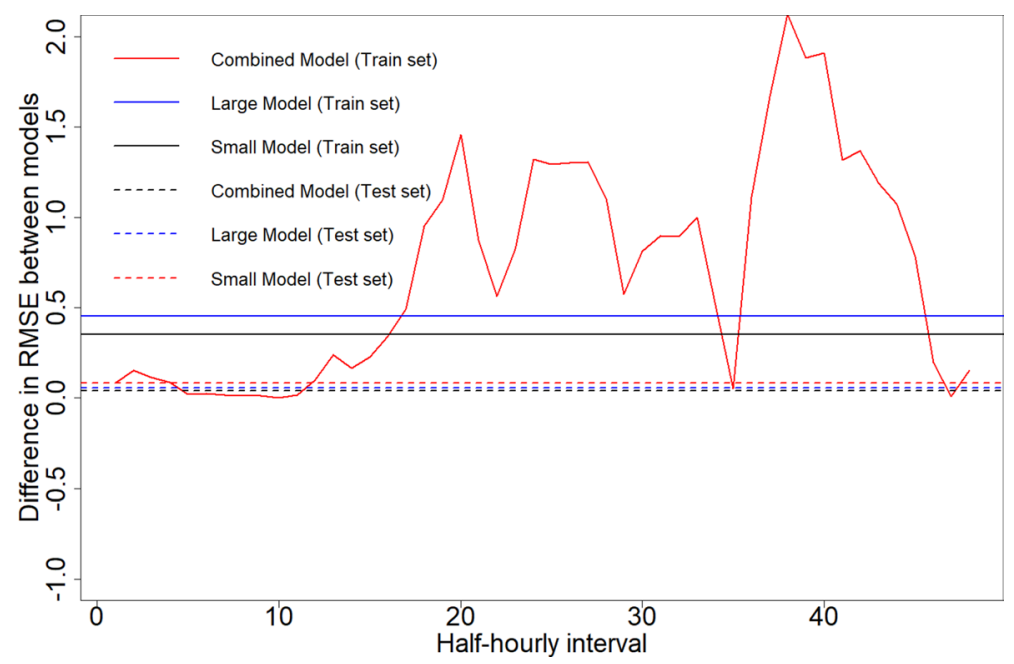

Fig. 3 Plot of the difference in RMSE between models with and without the price predictor 


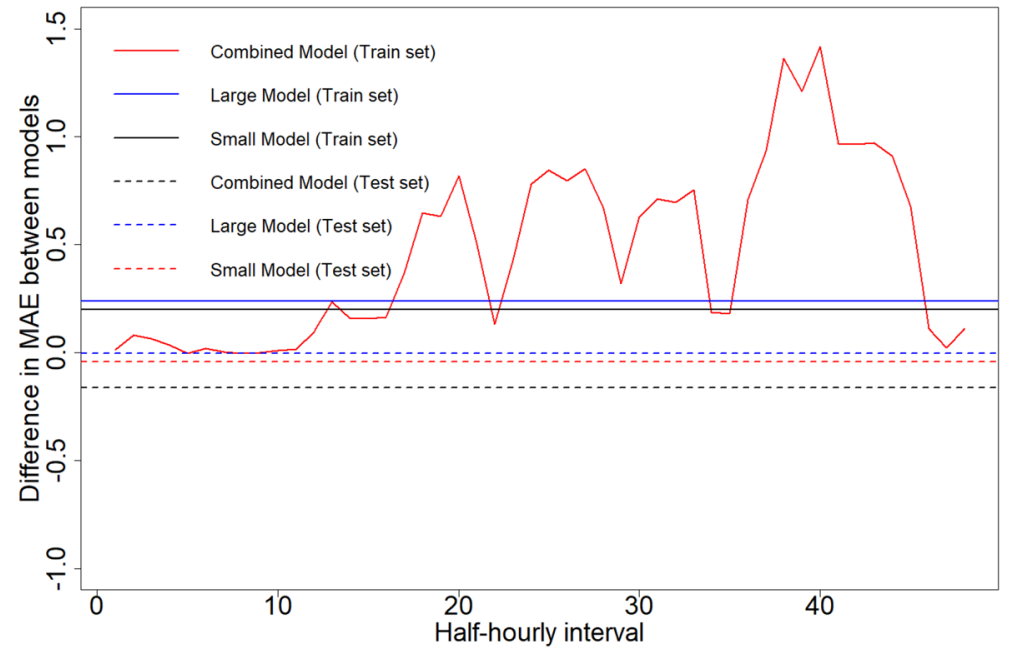

Fig. 4 Plot of the difference in MAE between models with and without the price predictor

The results show that the importance of the price predictor is low across all three models compared and some over fitting is apparent when comparing the respective performances between train and test sets. It is interesting to note that the only model which does not decrease its performance for neither RMSE and MAE nor train or test sets when the price predictor is dropped is the Large Model. Hence, we can conclude that the impact of the price predictor is low in the three models.

\subsubsection{Model selection}

Despite the results in the last section and considering that this low dependence on price probably stems from the shortcomings of the dataset exposed in Sect. 4.1, since choosing the best linear model possible is not the main focus of our work, we deem the price coefficient to be large enough for our purposes in all three models. Taking into consideration, now both the metrics and the importance of the price predictor exposed in Sect. 4.2.4 we conclude that the best fit for our work is the Large Model. It does not exhibit as much over fitting behaviour as the Combined Model, while being simpler and easier to work with. Moreover, it exhibits a better performance and higher impact for the price predictor with respect to the Small Model, while maintaining all its benefits aside from a lower number of variables which was not deemed sufficient to justify its selection. Thus, from this point forward, we consider only the Large Model and will be referred to simply as the linear model.

\subsection{Scenario generation and reduction}

For the stochastic problem dealt with in the following section, we require a scenario set to optimize over. To this end, we follow the procedure describe in 
Sect. 2.1 to generate a total of 1000 scenarios including random realization of pool prices $\bar{\lambda}_{t, \omega}^{P}$, availability of solar resources $A_{t, \omega}^{\mathrm{PPA}}$ and price coefficient predictors $\beta_{\omega}$. However, utilizing this raw scenario set can be computationally prohibitive. Thus, we seek to reduce it to a smaller subset of scenarios with similar statistical properties. To do this, we employ a scenario reduction technique proposed by Pineda and Conejo (2010) where the distance between scenarios is measured in terms of their impact on the problem objective function. Hence, we solve deterministic versions of problem (5) for each original scenario. Afterwards, mean shift clustering is utilized to group the scenarios into clusters with center $C_{k}$, finally the reduced scenarios are obtained by finding the closest scenario to the centers of these clusters, that is if $\mathcal{S}$ is the unreduced scenario set, $K$ is the number of clusters produced by the mean shift algorithm, $\mathcal{S}_{r}$ is the reduced scenario set and $f$ is the cost function given by the profit in (5):

$$
\mathcal{S}_{r}=\left\{s \in \mathcal{S} \mid \operatorname{argmin}\left\|f(s)-C_{k}\right\|_{2}, \quad k \in\{1, \ldots, K\}\right\}
$$

Once the reduced scenario set is produced, new probabilities have to be assigned to each reduced scenario by taking into account how many unreduced scenarios originally belonged to the cluster of the reduced scenario. This whole procedure is represented by the CDF's of the reduced and unreduced scenario sets of Fig. 5.

\subsection{Solution to the Stochastic problem}

We solve problem the stochastic problem (5) by means of the scenario set described in the previous section. Moreover, we consider that the maximum energy available for purchase from the forward and PPA contracts $\left(P_{\max }^{\mathrm{B}}\right.$ and $\left.P^{P P A}\right)$ is $80 \mathrm{kWh}$, at purchase prices of 4.6 and $4.8 \mathrm{p} / \mathrm{kWh}$, respectively. The mean value of the variable part of the price $\left(\bar{\lambda}^{E}\right)$ is assumed to be $1 \mathrm{p} / \mathrm{kWh}$. Bounds on the variable part of the price $\left(\lambda_{t}^{E}\right)$ are impose assuming $\gamma=0.25$ and the CVaR

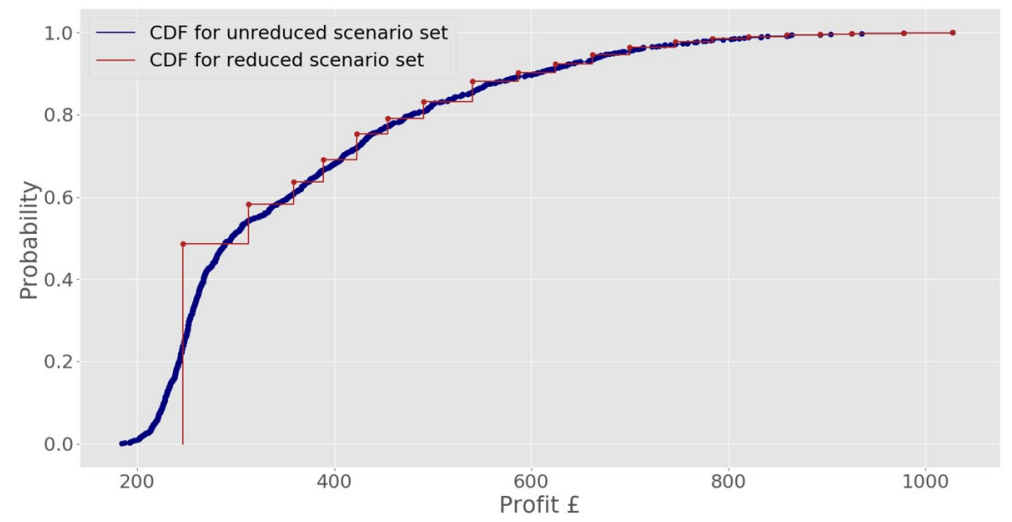

Fig. 5 CDF's of reduced and unreduced scenario sets 


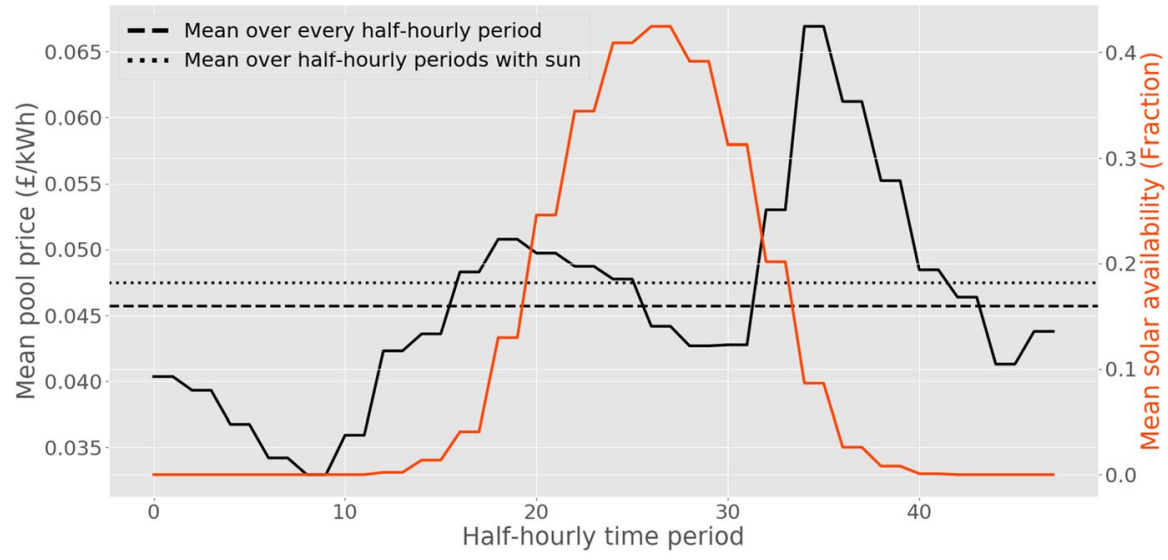

Fig. 6 Mean pool price and mean solar availability for the test month (December)

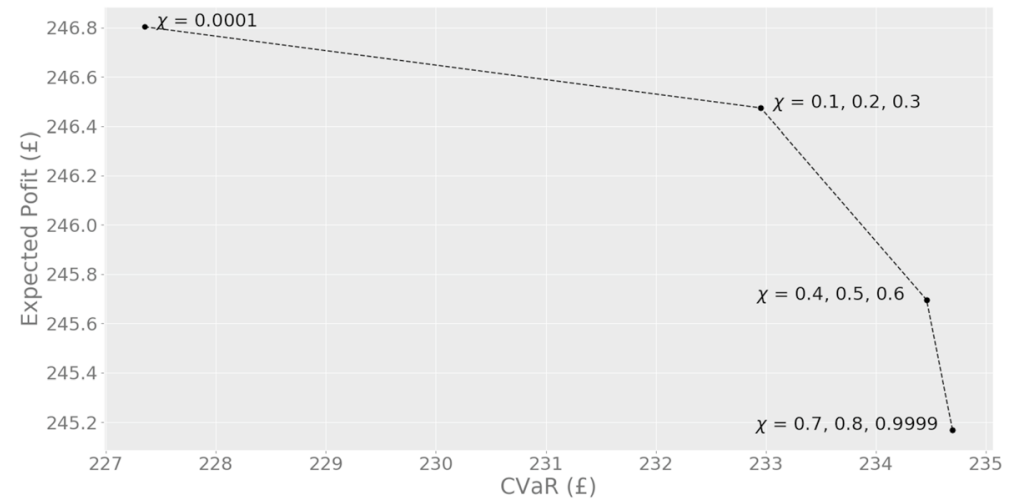

Fig. 7 Expected profit versus CVaR for different values of $\chi$. Problem was solved for values between 0 and 1 at intervals of 0.1 . This type of plot is called efficient frontier

is computed over the first 10-percentile of the profit distribution $(1-\alpha=0.9)$. Figure 6 depicts the summary (mean values) of the scenarios for pool prices and solar availability considered (generated as described in Appendix 1). The dotted line is the mean of pool prices over the half-hourly time periods which have a solar availability of at least 0.1 . Although this value was chosen arbitrarily, careful examination of the figure makes it clear that it will always be greater than the mean over all half-hourly time periods.

\subsubsection{Efficient frontier}

We are first interested in deciding which values of $\chi$ are meaningful to explore. For that purpose, we compare, for different values of $\chi$, the expected profit versus the CVaR. Note that values of $\chi=0$ (risk neutral) and $\chi=1$ (risk averse) are 


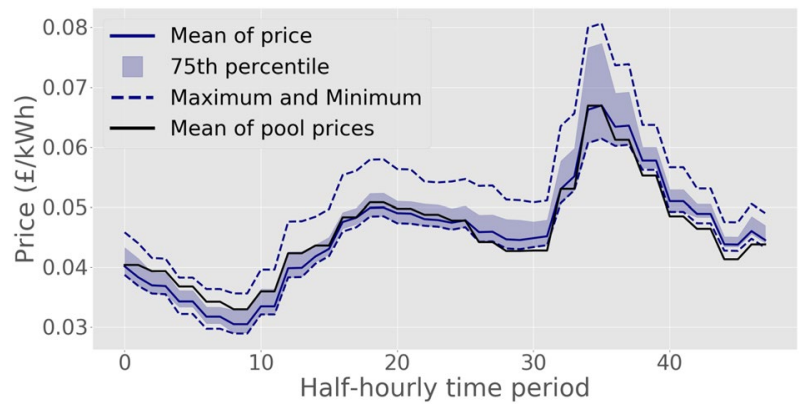

Fig. 8 Price distribution for $\chi=0.0001$ (risk-neutral)

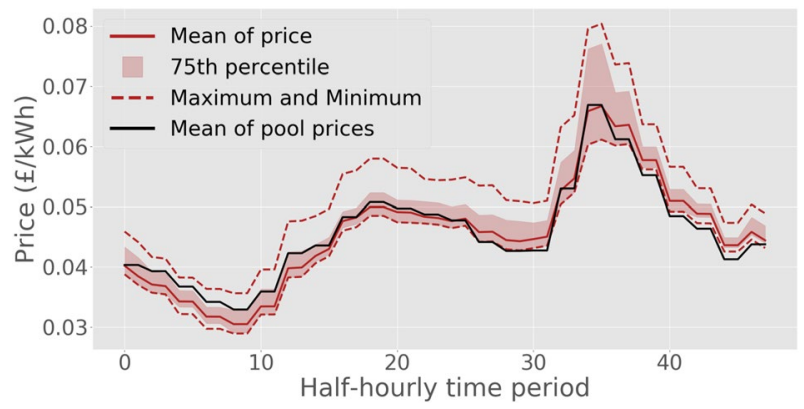

Fig. 9 Price distribution for $\chi=0.9999$ (risk-averse)

approximated by $\chi=0.0001$ and $\chi=0.9999$, respectively, to avoid numerical inconsistencies.

The resulting efficient frontier is depicted in Fig. 7, where we found four main groups of values of $\chi$ which entail different combination of expected profit vs CVaR. For clarity, in the following section, we focus our analysis on the two extreme values of $\chi=0.0001$ and $\chi=0.9999$. Also, it is interesting to note that the losses in expected profit are higly compensated by the increase of the CVaR, thus we can infer that the inclusion of risk for the retailer's optimal decision is probably desired, especially a value of $\chi$ between 0.1 and 0.3 since that is the region where the drop in expected profit is small with a large increased of the $\mathrm{CVaR}$.

\subsubsection{Daily price distribution}

We want to study how the prices offered by the retailer $\left(\lambda_{t, \omega}^{R}\right)$ behave at each hour and how these vary among scenarios. Figures 8 and 9 represent the distribution of prices for each day of the week in terms of their average half hourly values over the test set (month of December), for the risk neutral and risk averse cases, respectively. The maximum and minimum values are also included to quantify the level of price variability. 


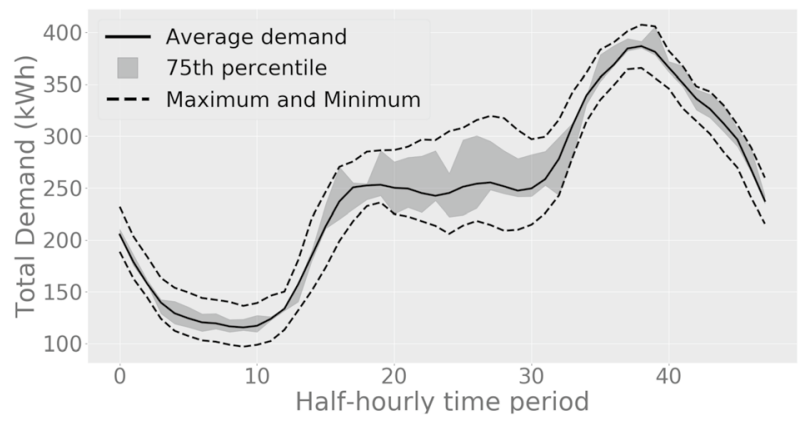

Fig. 10 Energy demand

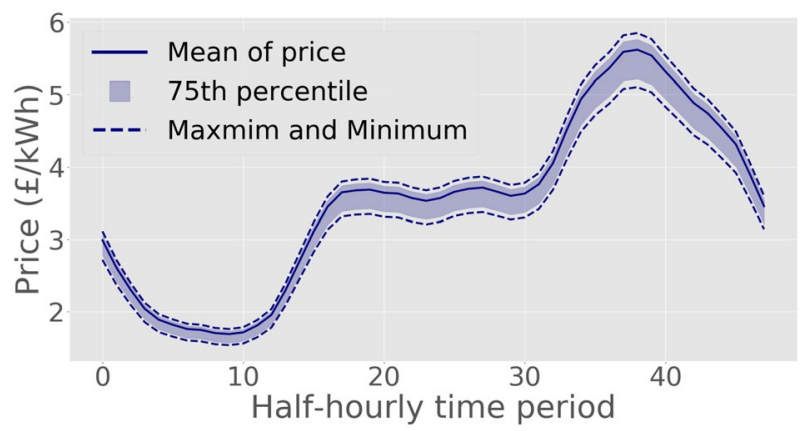

Fig. 11 Price distribution for $\chi=0.0001$ (risk-neutral), no direct constraints on retail prices

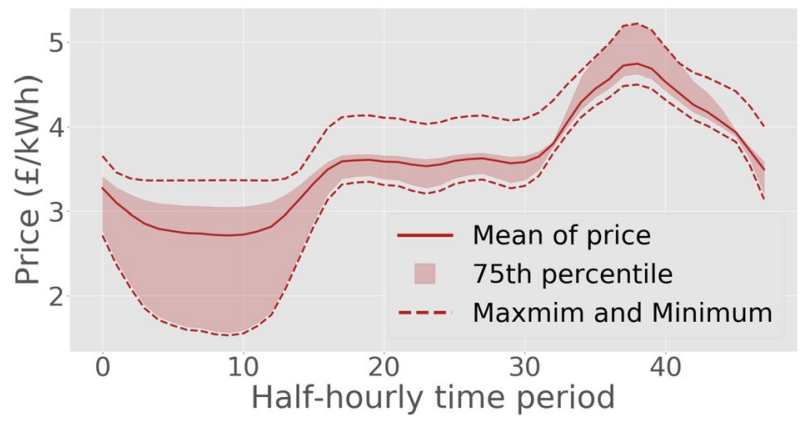

Fig. 12 Price distribution for $\chi=0.9999$ (risk-averse), no direct constraints on retail prices

Figures 8 and 9 show that the price distribution is the same for the two risk aversion levels considered. This is caused by the tight constrains imposed to control the price and indexing it to the pool price. Indeed, we can observe how the pool and the retail price follow analogous trajectories. Note that for some periods, the retailer is able to offer tariffs on average below the spot price. This is because part of its 
energy has been already purchased at fixed prices (below the spot price at many hours) through forward based or PPA contracts. The resulting total demand from the consumers (Fig. 10) is also the same for the two risk aversion levels considered. It follows the same trend that the retail price, as it linearly depends on it.

To exemplify this effect, we remove from the problem constraints (5i) and (5j), which represent market regulation to control retail prices, and we rerun the simulation to regenerate Figs. 11 and 12.

The prices are now unrealistically high as the retailer would have a very strong position in the market to raise prices (market power). However, an interesting results is that the retailer would have sufficient control over the prices for it to be an effective variable to control risk. This behaviour would be expected with groups of consumers with enough price-elasticity so that external price regulation (constraints) are no longer needed. This particular aspect is studied in detail in Sect. 4.4.5.

In the following, we analyze how risk is effectively controlled by the retailer through the appropriate involvement in the different types of contracts that are available.

\subsubsection{Profit distributions}

The profit cumulative distribution functions (CDFs) over the scenarios for the different values of $\chi$ are represented in Fig. 13 .

As expected, when risk aversion is considered, the left tail of the profit distribution moves to the right but the expected profit is reduced. However, it is interesting to see that a side effect of moving the left tail to the right also increases the profits in some of the scenarios to higher values than any of the profits of the distribution for $\chi=0.0001$. This is surprising because usually, when risk becomes a factor, the $\mathrm{CDF}$ of said distribution compresses over the $\mathrm{x}$ axis with respect to the CDF without risk considerations. Nonetheless, there is nothing preventing this behaviour from arising as the CVaR only restricts the left tail of the profit distribution.

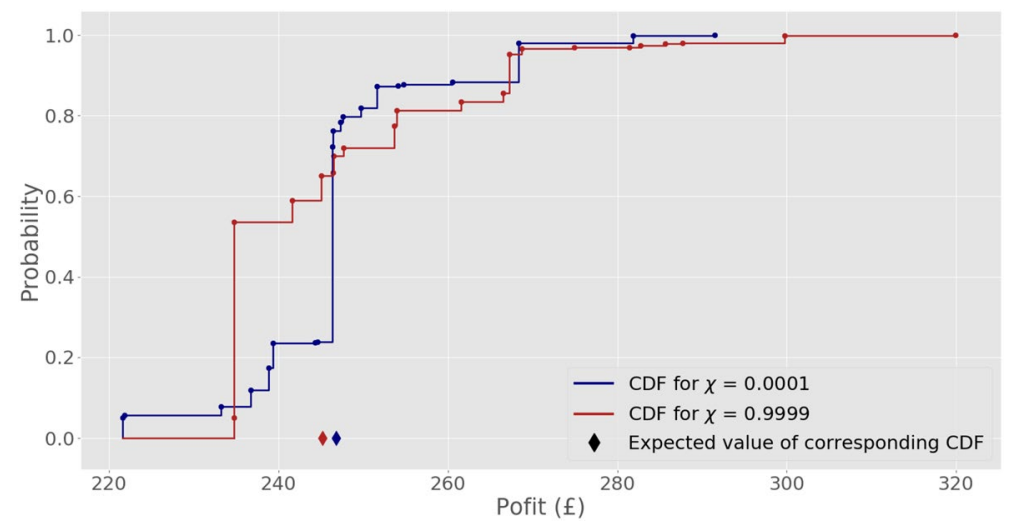

Fig. $13 \mathrm{CDF}$ of the profit distributions for $\chi=0.0001$ and $\chi=0.9999$ 


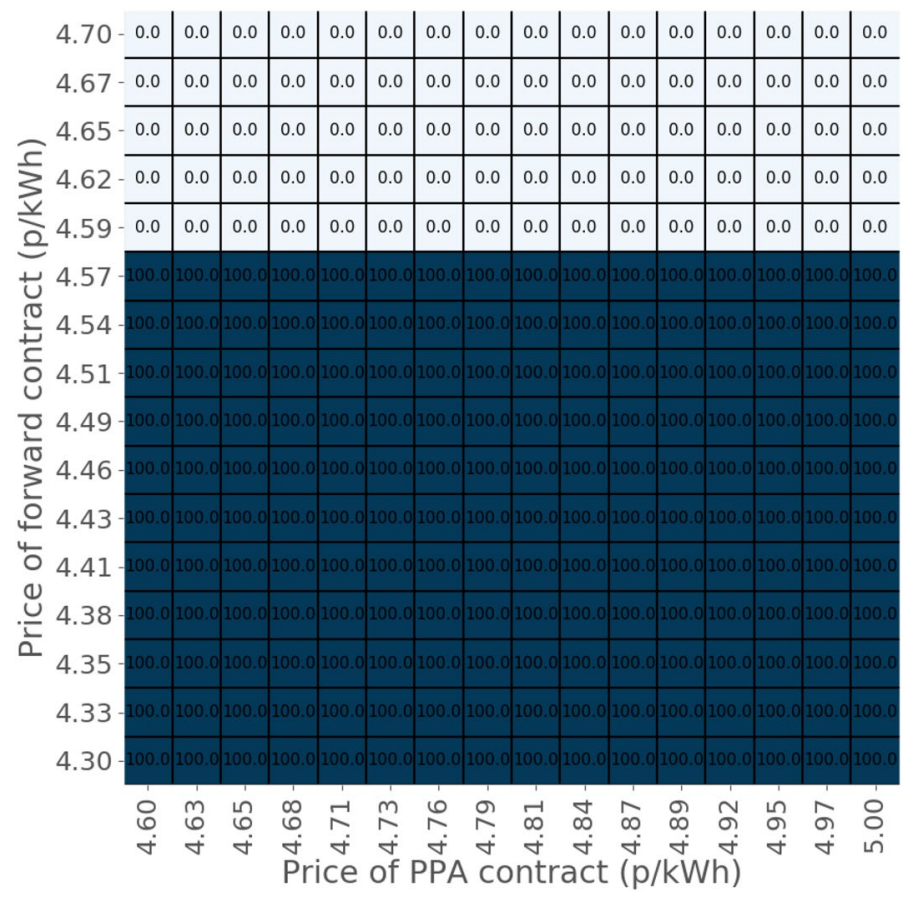

Fig. 14 Percentage of $p^{\mathrm{B}}$ contracted depending on prices of contracts for $\chi=0.0001$ (risk-neutral)

\subsubsection{Impact on contract prices}

We now investigate the decisions the retailer makes involving the amount of energy purchased from both the forward base contract and the PPA contract ( $p^{\mathrm{B}}$ and $\left.p^{\mathrm{PPA}}\right)$ depending on the prices of said contracts $\left(\bar{\lambda}^{\mathrm{B}}\right.$ and $\left.\bar{\lambda}^{\mathrm{PPA}}\right)$, and the risk aversion level. The results are presented in Figs. 14, 15, 16 and 17 as the percentage of energy bought with respect to the maximum available of the contract analyzed. Darker color means higher percentage. The actual percentage is represented within each cell.

For the risk neutral case (Fig. 14), the forward base contract $p^{\mathrm{B}}$ is only attractive if its price is below $4.59 \mathrm{p} / \mathrm{kWh}$. In this case, it is fully contracted regardless of the price offered by the renewable-based PPA contract $p^{\text {PPA }}$. Similarly, the PPA contract is signed at is maximum capacity if price is below $4.81 \mathrm{p} / \mathrm{kWh}$ (Fig. 15), regardless of the price offered by the forward base contract. This threshold price is above the previous one, because the PPA contract with a solar PV producer provides energy to the retailer in the central part of the day, which has associated higher pool prices (see Fig. 6). On the other hand, the forward contract is only competitive at lower prices, because is a base contract which provides energy in every period of the day, including night periods with low pool prices.

In the risk averse case, the forward base contract $p^{\mathrm{B}}$ (Fig. 16) is signed at its maximum capacity if its price is below $4.43 \mathrm{p} / \mathrm{kWh}$, which is below the price-threshold observed in the risk neutral case. However, for prices above this value, this contract 


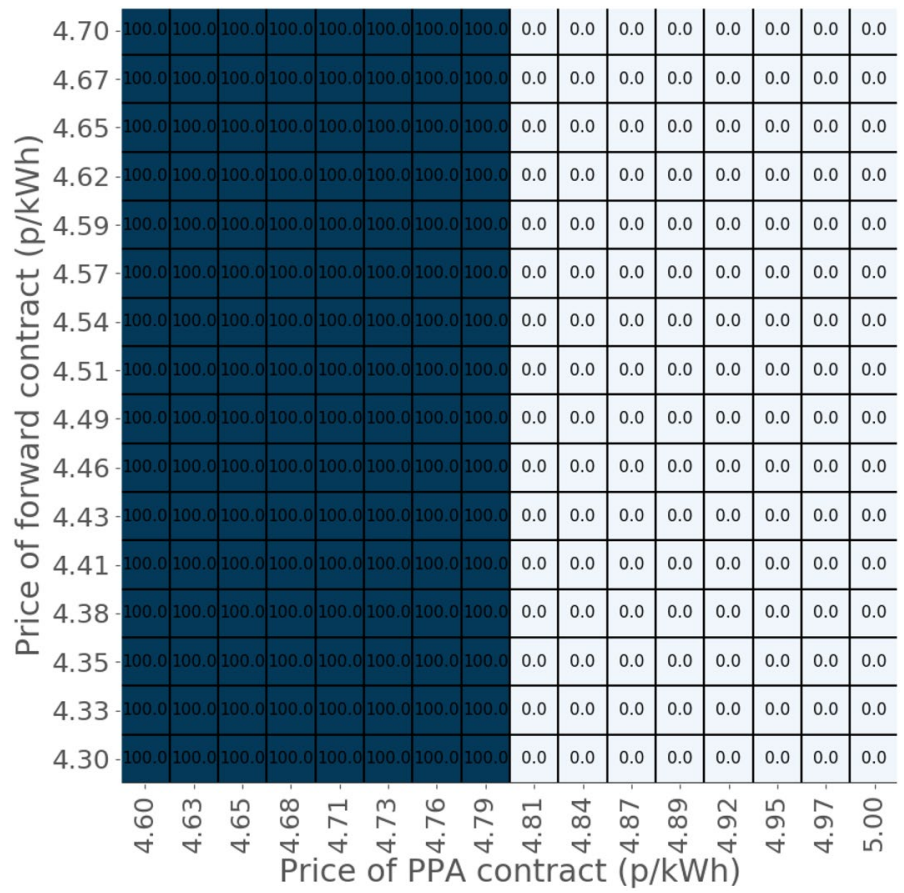

Fig. 15 Percentage of $p^{\mathrm{PPA}}$ contracted depending on prices of contracts for $\chi=0.0001$ (risk-neutral)

is also partially contracted if the prices of the PPA are relatively high. Similarly, the PPA contract (Fig. 17) is signed at is maximum capacity if its price below $4.63 \mathrm{p} /$ $\mathrm{kWh}$, which also below the price-threshold observed in the risk neutral case. We can also observe that for prices above $4.63 \mathrm{p} / \mathrm{kWh}$, the PPA is also contracted (partially) when the forward contract price is high. This diversification strategy, which is characteristic of risk averse settings, is more present for PPA contracting, as it introduces further levels of uncertainty due to its dependency on the renewable source. Overall, the risk-averse forward and PPA contracting volumes are decreased and used only if the prices are sufficiently attractive (small). This strategy helps avoiding potential unfavourable scenarios where forward contracting may exceed the demand realization, and hence incur in low profits.

\subsubsection{Effects of shifts on the mean of the price coefficient distribution}

We now tackle the effects of modifying the price coefficient to simulate more sensitive costumers to price signals. We seek to recreate the actual tendency of electrical systems where consumers are becoming more and more elastic to prices thanks to the technological advances provided within the smart grid paradigm (smart meters, electric vehicles and local electricity storage, intelligent appliances, distributed generation, etc.), and its impact on retailers' strategies. 


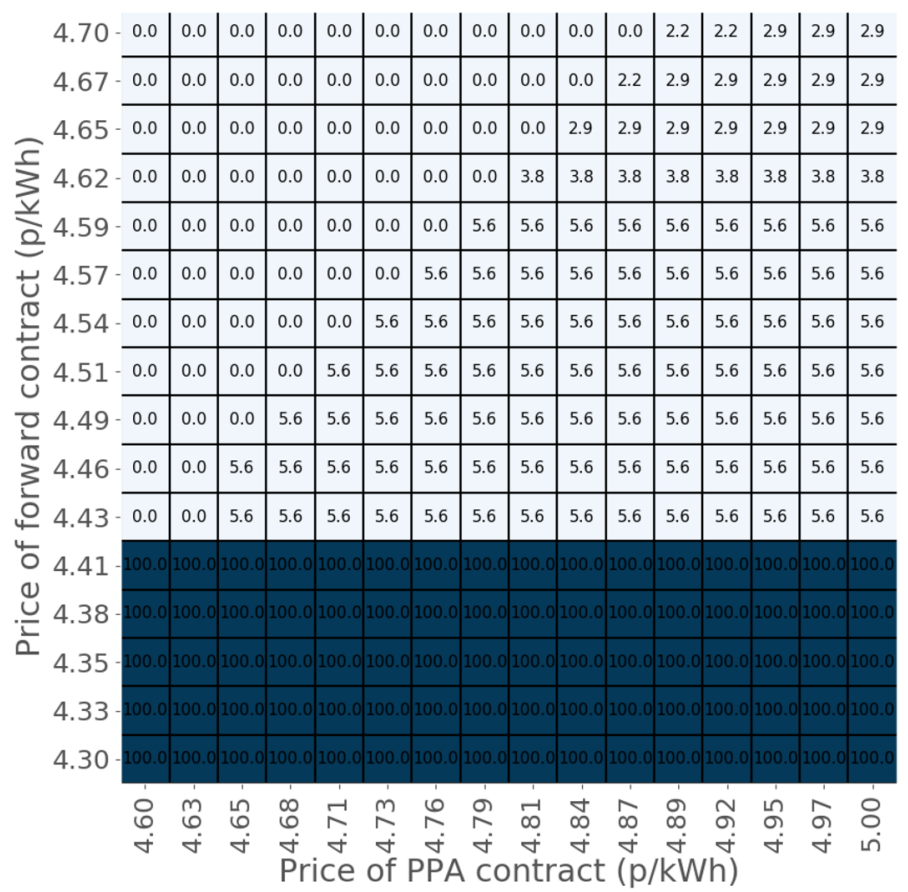

Fig. 16 Percentage of $p^{\mathrm{B}}$ contracted depending on prices of contracts for $\chi=0.9999$ (risk-averse)

For this purpose, we modify the distribution of $\beta_{1}$ (assuming again this represents the price coefficient) given by (4) by adding a shift term $\beta_{\text {shift }}$ as in:

$$
\hat{\beta}_{1} \rightarrow \mathcal{N}\left(\mathbb{E}\left[\hat{\beta}_{1}\right]-\beta_{\text {shift }}, \sigma_{\epsilon}^{2} \sum_{j=1}^{n}\left[\left(\mathbf{X}^{T} \mathbf{X}\right)^{-1} \mathbf{X}^{T}\right]_{1 j}^{2}\right)
$$

Note that the variance of the distribution is kept the same and only the mean is shifted. This would be equivalent to having a hypothetical dataset with a greater impact of the price coefficient while maintaining the accuracy of the estimation of the coefficient's distribution. We now consider two effects, the impact on the average price (8), taken to be a scalar summary of the price distribution, and the impact on the profit distribution over the scenario set.

$$
\text { average price }=\frac{1}{|T|} \sum_{t \in T} \sum_{\omega \in \Omega} \pi_{\omega} \lambda_{t, \omega}^{\mathrm{R}}
$$

The first result is that the impact on the average price as a function of $\beta_{\text {shift }}$ under the current formulation (5) is almost negligible, as it remains constant at a value of for both risk-neutral and risk-averse cases of $4.56 \mathrm{p} / \mathrm{kWh}$. This is caused by the tight constraints that regulate the retail price. However, the effects on the profit distribution are more interesting and are represented as violin plots in Fig. 18. It is clear 


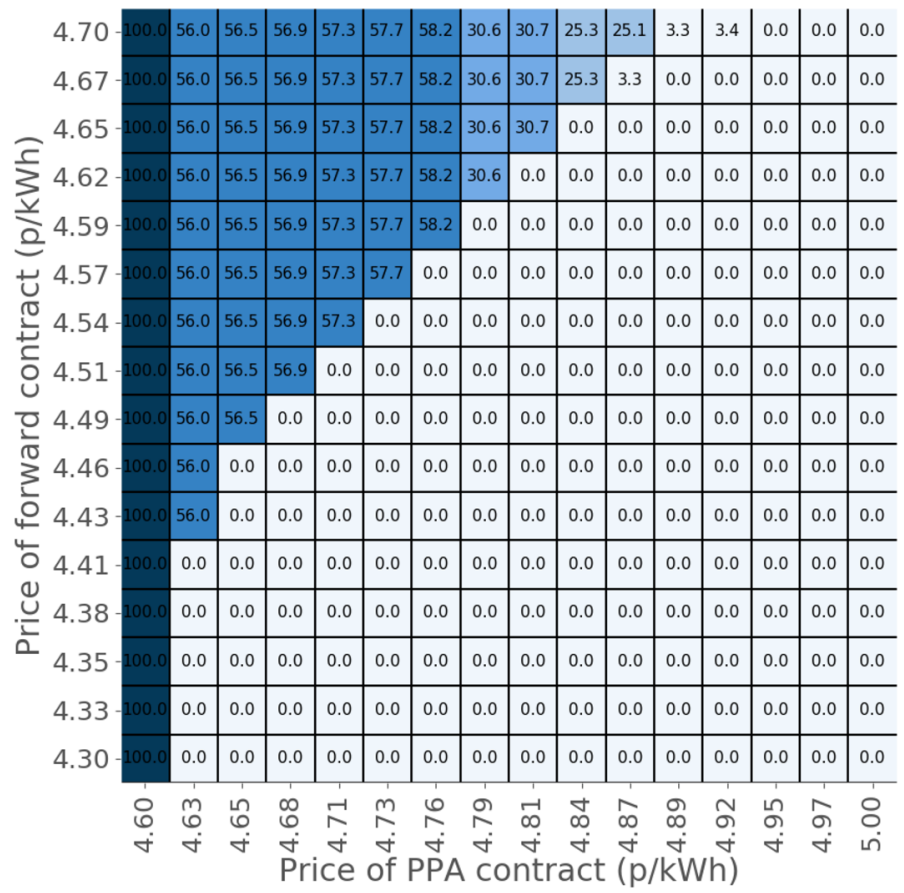

Fig. 17 Percentage of $p^{\mathrm{PPA}}$ contracted depending on prices of contracts for $\chi=0.9999$ (risk-averse)

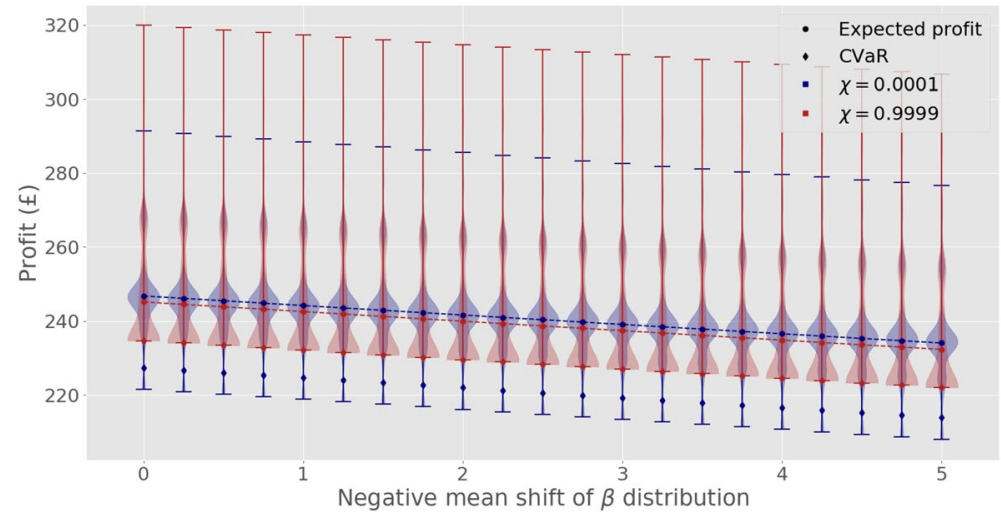

Fig. 18 Violin plots showing profit distributions for both values of $\chi$

that as $\beta_{\text {shift }}$ increases (higher price-sensitivity of consumers), the expected profit decreases, albeit slightly. On the other hand, the distribution does not change as a function of $\beta_{\text {shift }}$ on this problem, as retail prices do not vary the main cause for this decrease is a reduction in the overall consumers demand. 


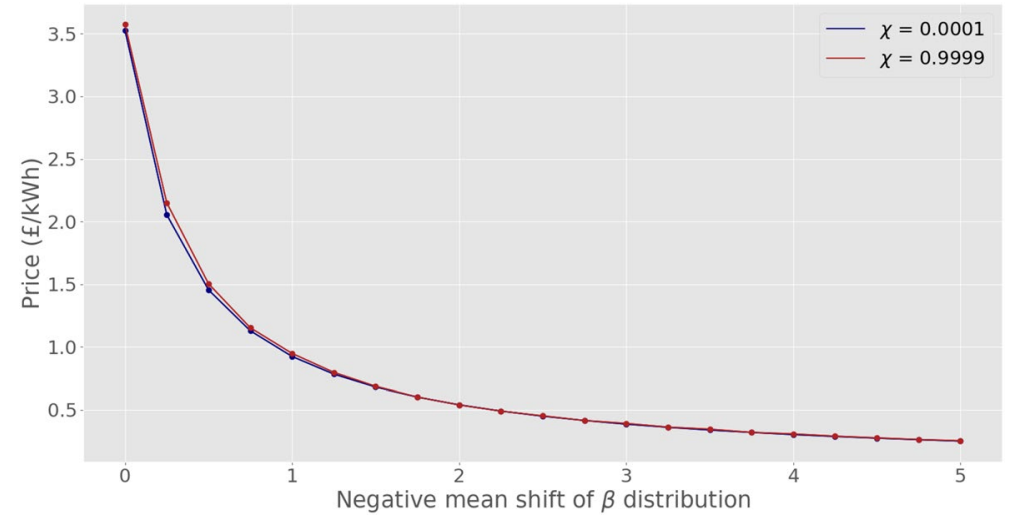

Fig. 19 Plot of average price for both values of $\chi$

However, now that we are essentially increasing the impact of price-demand sensitivity $\left(\beta_{1}\right)$, we can explore if for larger values of this parameter the retail price can be self-regulated by consumers and no external market rules are needed, i.e., verify if constraints (5i)-(5k) could be dropped from the model. For this purpose, we first analyze for which values of $\beta_{\text {shift }}$ the self-regulation of prices is sufficient (keeping in mind that $\mathbb{E}\left[\hat{\beta}_{1}\right]=-0.36$ ). We also choose a 'reasonable' boundary for the price by considering the mean of the actual pool prices from the dataset, which is $13.6 \mathrm{p} /$ $\mathrm{kWh}$, and by assuming that prices has to be lower than double this price.

Figure 19 represents how the average retail price varies with $\beta_{\text {shift }}$ for risk-neutral and risk-averse retailers when solving problem (5) where the price-regulating constraints (5i)-(5k) are dropped. We see how retail prices self-regulate as the impact of $\beta_{1}$ increases (higher degree of price-sensitivity by consumers). Note that prices start being "reasonable" around a value of $\beta_{\text {shift }} \approx 4.75$.

Having identified appropriate values of $\beta_{\text {shift }}$ to self regulate the retail prices, we now explore the profit distribution for the problem with Eqs. (5i)-(5k) dropped. As can be seen in Fig. 20 the expected profits exhibit a similar behaviour to the retail prices in Fig. 19. However, the behaviour is much richer than that of Fig. 18 as not only does the profit vary non-linearly, but, as can be seen in Figs. 20b, c the profit distribution also varies (we illustrate the cases where $\beta_{\text {shift }}$ equals $0,4.75$ and 5). It might be tempting to compare the profits from Figs. 20 to those of 18, however, the average price for $\beta_{\text {shift }}=5$ is still around $25 \mathrm{p} / \mathrm{kWh}$ (now it does depend on $\chi$ ) while, as mentioned previously, average price for the restricted problem was $4.56 \mathrm{p} / \mathrm{kWh}$. To mimic this price, $\beta_{\text {shift }}$ must be increased all the way up to 52 where the average price differs now in less than $1 \%$. The results of increasing $\beta_{\text {shift }}$ to 52 are shown in Table 2, implying that the

Table 2 Expected profits in pounds for the different problems and the different values of $\chi$

\begin{tabular}{lll}
\hline & $\chi=0$ & $\chi=1$ \\
\hline Free problem & 530.34 & 514.01 \\
Restricted problem & 137.64 & 132.54 \\
\hline
\end{tabular}




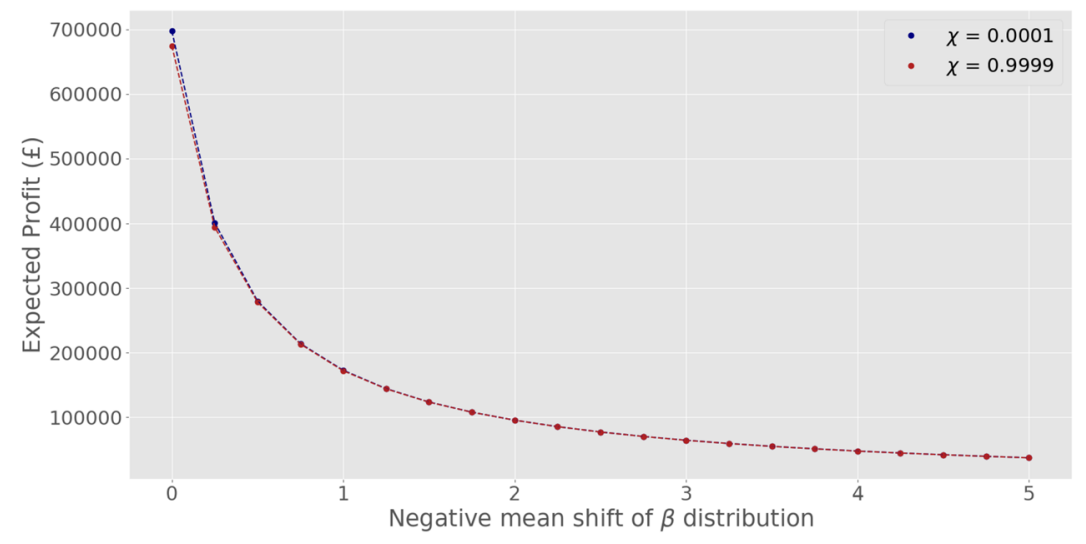

(a)

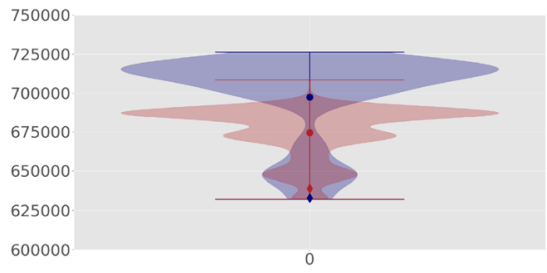

(b)

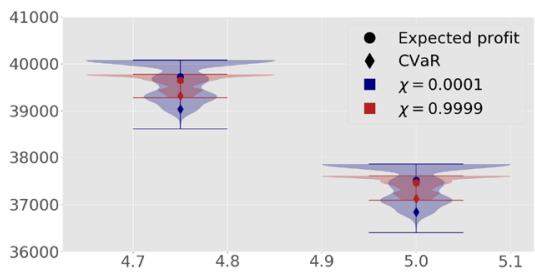

(c)

Fig. 20 Expected profit as a function of $\beta_{\text {shift }}(\mathbf{a})$. Violin plot for $\beta_{\text {shift }}=0(\mathbf{b})$, and for $\beta_{\text {shift }}=4.75$ and $\beta_{\text {shift }}=5(\mathbf{c})$

problem without restrictions performs much better for the retailer as the profits reached are much higher than those of $\beta_{\text {shift }}=0$ for the restricted problem.

\section{Closing remarks and future work}

Although the dataset has been a limiting factor throughout this whole work because of being only slightly dependent on price, we have managed to formulate a restricted problem which could be adapted to these hypothetical low-sensitivity customers which produces both highly competitive prices and safe profits for the retailer. We have also successfully included both stochasticity surging from data-driven scenario generation and the $\mathrm{CVaR}$ as a risk measure to increase the retailer's profit in the worst-case scenarios. Nonetheless, we believe that this low impact of the price variable stems only from the scarce options offered to retailers in the ToU tariff implemented in London in 2013 and that future datasets produced with more price options (or continuous prices) would not need these restrictions to formulate an economically viable problem. For this reason, we have artificially increased the sensitivity of customers to construct a simpler 
problem with fewer restrictions as the price can now be regulated only by means of the price sensitivity. This produces a much richer problem, both in expected profit for the retailer and in theoretical interest.

The numerical results obtained from the case study indicate that risk hedging can be efficiently performed through the energy procurement strategy of the retailer. In this manner, the profit in worst scenarios can be increased at expenses of reducing slightly the total expected profit. However, the selling price offered to the clients is not influenced by the risk-aversion degree of the retailer in the analyzed case. It is interesting to note that the forward (base) contract is signed for a contract price smaller than that associated with the PPA contract in the riskneutral case. In other words, the retailer is willing to pay a higher price for a PPA contract with uncertain production if this contract allows it to reduce the purchase of energy in pool during the central periods of the day, which are characterized by higher pool prices. In this case, forward and PPA contracts are not signed for prices higher than 4.57 and $4.79 \mathrm{p} / \mathrm{kWh}$, respectively. However, if risk is accounted for, the retailer purchases small amounts of energy from forward and PPA contracts even for high prices, up to 4.70 and $4.92 \mathrm{p} / \mathrm{kWh}$ for forward and PPA contracts, respectively.

It has been also observed that the price offered by the retailer is highly dependent on sensitivity of the costumers to prices. Assuming that the switch of electricity supplier by consumers is not considered in this model, a low price elasticity may lead to unreasonable results if the selling price offered by the retailer is not constrained. However, if the price elasticity of consumers is high enough, the retail price is reasonable even though this price is not bounded.

Future work is focused on considering simultaneously the sensitivity of consumers to prices and the possibility of switching supplier. Additionally, more complex price tariffs may be investigated to increase the profitability of the retailer under price-sensitive consumers.

\section{Appendix}

\section{Heuristic argument for the usage of the Hajek-Sidak CLT}

Theorem 1 (Hajek-Sidak Central Limit Theorem, Ma et al. 2020) Let $X_{1}, \ldots, X_{n}$ be independent and identically distributed (i.i.d.) random variables such that $\mathbb{E}\left[X_{i}\right]=\mu$ and $\operatorname{Var}\left[X_{i}\right]=\sigma^{2}$ are both finite. Define $T n=d_{1} X_{1}+\cdots+d_{n} X_{n}$, then:

$$
\frac{T_{n}-\mu \sum d_{i}}{\sigma \sqrt{\sum d_{i}^{2}}} \rightarrow \mathcal{N}(0,1)
$$

whenever the Noether condition: 


$$
\frac{\max _{1 \leq i \leq n} d_{i}^{2}}{\sum_{i=1}^{n} d_{i}^{2}} \rightarrow 0, \quad \text { as } \quad n \rightarrow \infty
$$

is satisfied.

This theorem implies that the normal approximation used is valid as long as the entries of the matrix $n^{-1}\left(\mathbf{X}^{T} \mathbf{X}\right)$ can be bounded from above and below as that implies that $\max _{1 \leq i \leq n} d_{i}^{2}=\max _{1 \leq i \leq n}\left[n^{-1}\left(\mathbf{X}^{T} \mathbf{X}\right)^{-1} \mathbf{X}^{T}\right]_{i j}^{2}$ must also be bounded while the bound from below ensures that when $n \rightarrow \infty, \sum_{i=1}^{n} d_{i}^{2}=\sum_{j=1}^{n}\left[n^{-1}\left(\mathbf{X}^{T} \mathbf{X}\right)^{-1} \mathbf{X}^{T}\right]_{i j}^{2}$ diverges for being an infinite sum of a positive non-zero constant, implying that the Noether condition is satisfied. To bound the entries of the matrix $n^{-1}\left(\mathbf{X}^{T} \mathbf{X}\right)$ its eigenvalues are used. We now prove the upper bound for the matrix entries given that an upper bound for the maximum eigenvalue of $n^{-1}\left(\mathbf{X}^{T} \mathbf{X}\right)$ exists.

Theorem 2 Let $\lambda_{\max }<\infty$ be the maximum eigenvalue of a given unitarily diagonalizable matrix $\mathbf{A} \in \mathbb{R}^{k \times k}$, then $\left|\mathbf{A}_{i j}\right| \leq \kappa$ where $\kappa$ is some undetermined constant.

Proof Since $\mathbf{A}$ is unitarily diagonalizable, $\mathbf{A}=\mathbf{U} \mathbf{\Lambda} \mathbf{U}^{T}$ and using the frobenius norm:

$$
\|\mathbf{A}\|_{F}=\sqrt{\sum_{i=1}^{k} \sum_{j=1}^{k}\left|\mathbf{A}_{i j}\right|^{2}}=\left\|\mathbf{U} \mathbf{\Lambda} \mathbf{U}^{T}\right\|_{F}=\|\mathbf{\Lambda}\|_{F}=\sqrt{\sum_{i=1}^{k}\left|\lambda_{i}\right|^{2}} \leq \sqrt{k} \lambda_{\max }<\infty
$$

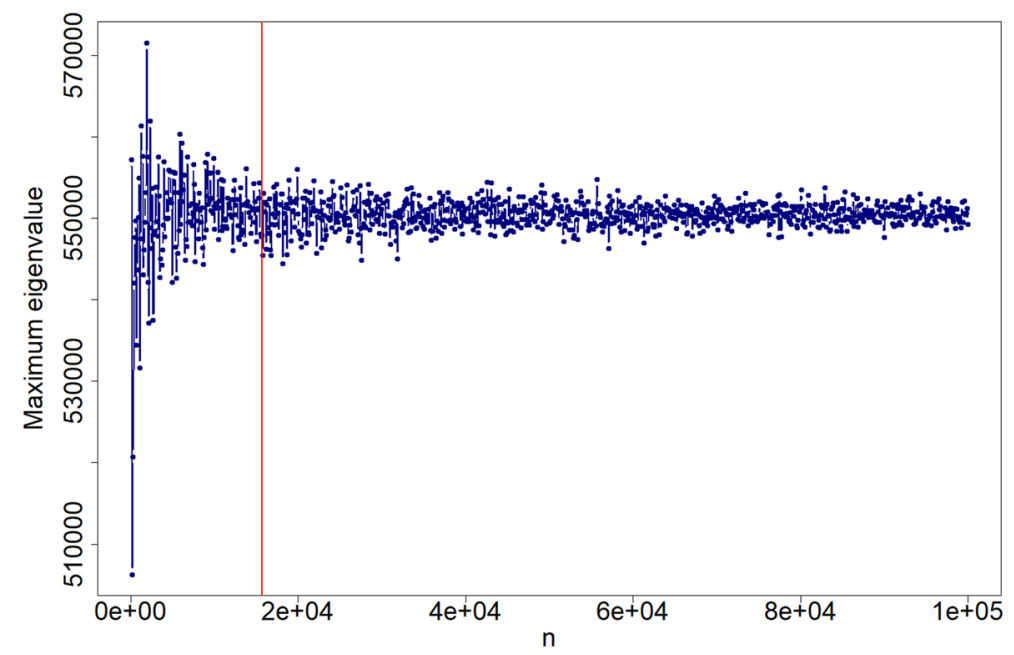

Fig. 21 Plot of maximum eigenvalue as $n$ increases. The red line indicates the actual size of the matrix $\mathbf{X}$ 


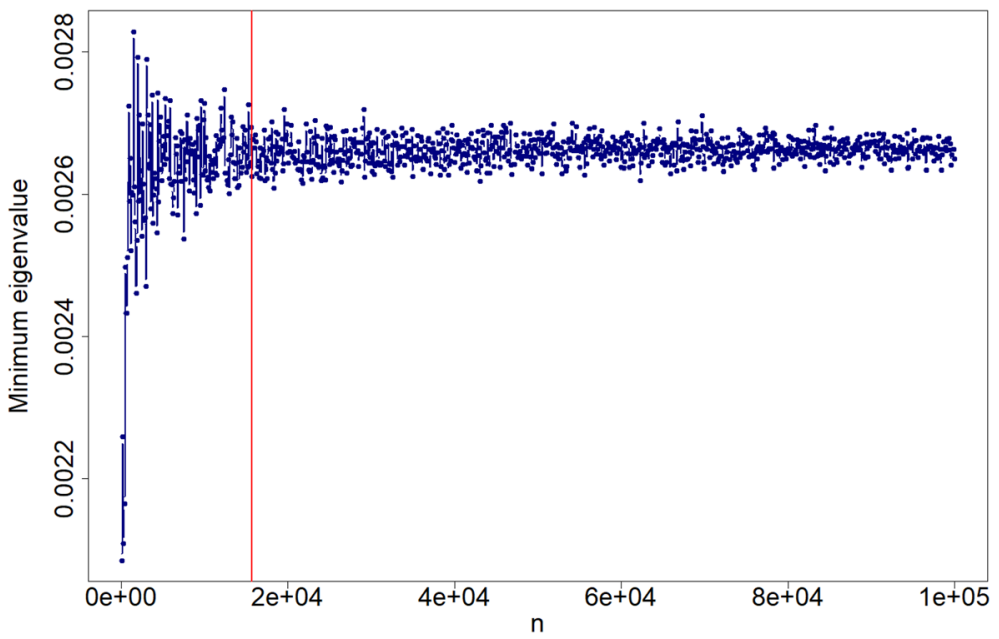

Fig. 22 Plot of minimum eigenvalue as $n$ increases. The red line indicates the actual size of the matrix $\mathbf{X}$

Theorem 3 Let $\lambda_{\min }>0$ be the minimum eigenvalue of a given unitarily diagonalizable matrix $\mathbf{A} \in \mathbb{R}^{k \times k}$, then $\left|\mathbf{A}_{i j}\right|>0$.

Proof

$$
\|\mathbf{A}\|_{F}=\sqrt{\sum_{i=1}^{k} \sum_{j=1}^{k}\left|\mathbf{A}_{i j}\right|^{2}}=\left\|\mathbf{U} \mathbf{\Lambda} \mathbf{U}^{T}\right\|_{F}=\|\boldsymbol{\Lambda}\|_{F}=\sqrt{\sum_{i=1}^{k}\left|\lambda_{i}\right|^{2}} \geq \sqrt{k} \lambda_{\min }>0
$$

Since the matrix $n^{-1}\left(\mathbf{X}^{T} \mathbf{X}\right)$ is a normal matrix, it is also unitarily diagonalizable, therefore its entries can be bounded if an upper and lower bound for its eigenvalues is found. Proofs for bounds of eigenvalues of real data matrices are hard to produce and thus this argument is purely heuristic. As can be seen in the following figures, as we increase $n$ in our data matrix by resampling the matrix's rows via bootstrap, both the maximum and minimum eigenvalue appear to converge to a given value (Figs. 21, 22).

These results are considered sufficient for the application of the Hajek-Sidak CLT to the present data matrix.

\section{Scenario generation for pool prices and solar availability}

To generate scenarios for both the pool prices and the solar availability we need a method which allows the generation of a large number of scenarios, as this set will be reduced later by other methods. Thus the following method is proposed. The appropriate data (either the pool price data spanning the years 2013-2020 or the solar availability data spanning the years 2010-2019) is split into a training, 


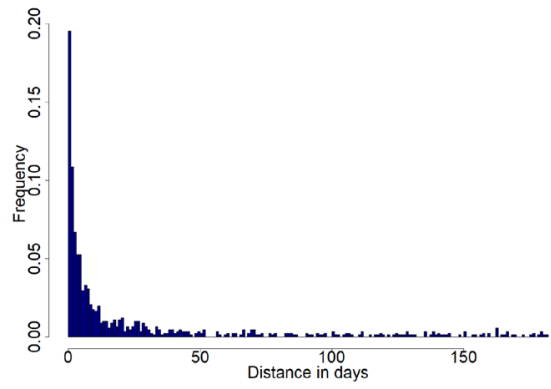

(a) Distribution of distance in days

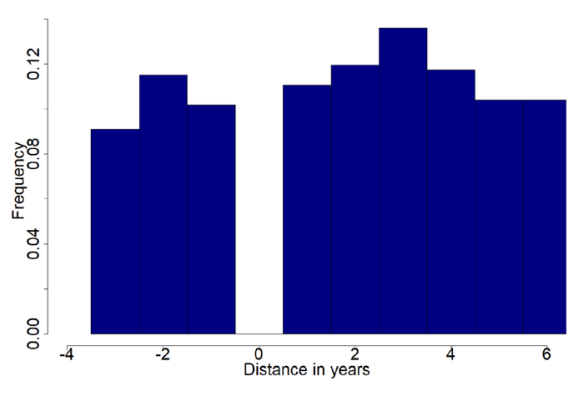

(b) Distribution of distance in years

Fig. 23 Distributions for the solar availability data. Since 2013 is excluded from the comparison set, a distance of 0 years is impossible

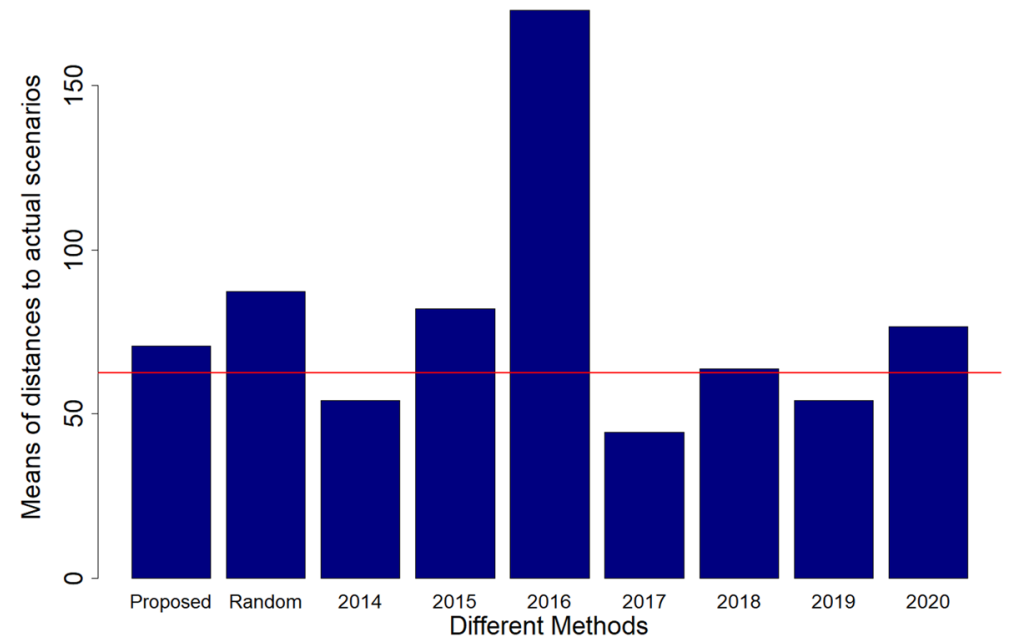

Fig. 24 Barplot comparing the different methods for the pool prices data. The red line is the average of the years 2014 through 2020 except 2016 given its anomalous behaviour

validation, test and comparison sets, the validation set being November 2013, the test set being December 2013 as in the rest of the present work, the training set being every date in the year 2013 excluding those in other sets and the comparison set being every date of every other year. For each date in the training set, the three closest dates from the comparison set are found by minimizing the euclidean norm between their respective scenarios. From these dates two separate empirical distributions of distance in days and distance in years are found. These distributions are exemplified in Fig. 23 for the solar availability data.

Finally, by sampling from these distributions, scenarios are generated for each date in the validation set by taking the corresponding scenario to the sampled date. In Figs. 24 and 25 a comparison between this proposed method, completely random sampling and same date selection from different years is made for both the pool 


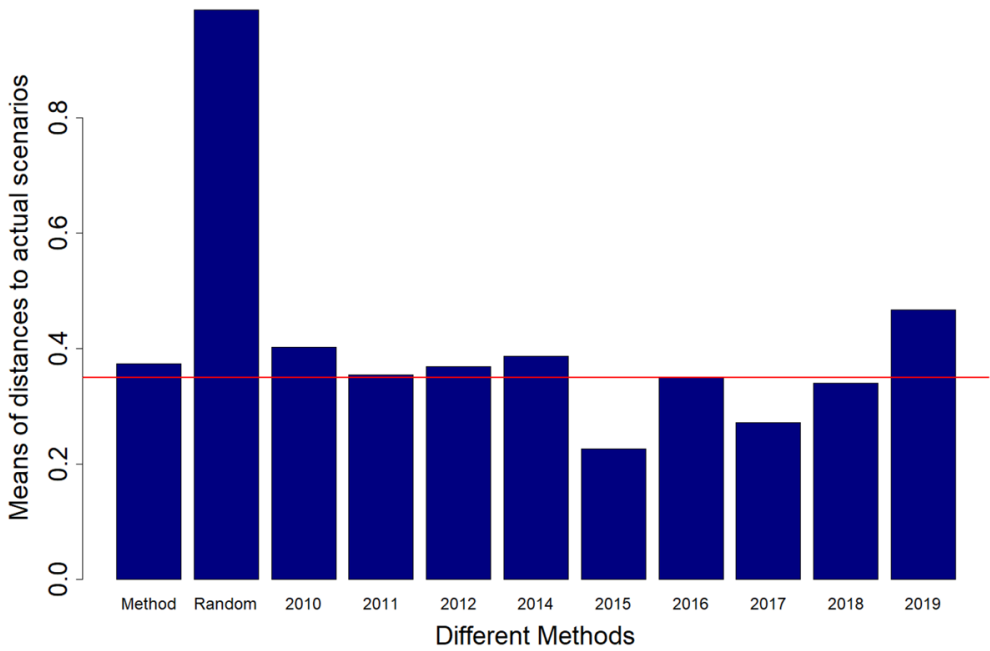

Fig. 25 Barplot comparing the different methods for the solar availability data. The red line is the average of the years 2010 through 2019 except 2013 given that its the year used to compare to

prices and solar availability data. Bars represent an average of euclidean distance between actual realizations for the validation set and the generated scenario.

As can be seen in these figures, while most years perform better than the proposed method, in particular their average, which could also be used to generate scenarios, this type of procedure is limited in the amount of scenarios it can produce (in this case it can only produce a maximum of 6 scenarios for each date for the pool price data and a maximum of 9 scenarios for the solar availability data). This, however, is not a problem for the proposed method which can handle far larger numbers of scenarios while performing noticeably better than the random method. This is why this approach is taken for the generation of pool price scenarios. Finally, the validation set is included in the training set and scenarios are generated for the test set.

Acknowledgements The authors gratefully acknowledge the financial support from the Spanish government through projects PID2020-116694GB-I00 and PID2019-111211RBI00/ AEI/10.13039/501100011033, and from the Madrid Government (Comunidad de Madrid) under the Multiannual Agreement with UC3M in the line of "Fostering Young Doctors Research" (ZEROGASPAIN-CM-UC3M), and in the context of the V PRICIT (Regional Programme of Research and Technological Innovation.

Funding Open Access funding provided thanks to the CRUE-CSIC agreement with Springer Nature.

Open Access This article is licensed under a Creative Commons Attribution 4.0 International License, which permits use, sharing, adaptation, distribution and reproduction in any medium or format, as long as you give appropriate credit to the original author(s) and the source, provide a link to the Creative Commons licence, and indicate if changes were made. The images or other third party material in this article are included in the article's Creative Commons licence, unless indicated otherwise in a credit line to the material. If material is not included in the article's Creative Commons licence and your intended use is not permitted by statutory regulation or exceeds the permitted use, you will need to obtain permission 
directly from the copyright holder. To view a copy of this licence, visit http://creativecommons.org/licen ses/by/4.0/.

\section{References}

Akhavan-Hejazi H, Mohsenian-Rad H (2018) Power systems big data analytics: an assessment of paradigm shift barriers and prospects. Energy Rep 4:91-100

Carrión M, Conejo AJ, Arroyo JM (2007) Forward contracting and selling price determination for a retailer. IEEE Trans Power Syst 22(4):2105-2114

Carrión M, Conejo AJ, Arroyo JM (2007) A bilevel stochastic programming approach for retailer futures market trading. IEEE Trans Power Syst 24(3):1446-1456

Conejo AJ, Carrión M, Morales JM (2010) Decision making under uncertainty in electricity markets. Springer

Deng T, Yan W, Nojavan S, Jermsittiparsert K (2020) Risk evaluation and retail electricity pricing using downside risk constraints method. Energy 192:116672

Dong S, Li H, Wallin F, Avelin A, Zhang Q, Yu Z (2019) Volatility of electricity price in Denmark and Sweden. Energy Proc 4331-4337:2019

European Commission (2021) EU emissions trading system. https://ec.europa.eu/clima/policies/ets_en

Fan S, Hyndman RJ (2012) Short-term load forecasting based on a semi-parametric additive model. IEEE Trans Power Syst 27(1):134-141

Feng C, Wang Y, Zheng K, Chen Q (2020) Smart meter data-driven customizing price design for retailers. IEEE Trans Smart Grid 11(3):2043-2054

García-Bertrand R (2013) Sale prices setting tool for retailers. IEEE Trans Smart Grid 4(4):2028-2035

Hatami A, Seifi H, Sheikh-El-Eslami MK (2011) A stochastic-based decision-making framework for an electricity retailer: time-of-use pricing and electricity portfolio optimization. IEEE Trans Power Syst 26(4):1808-1816

Hyndman RJ, Athanasopoulos G (2018) Forecasting: principles and practice, 2nd edition. OTexts.com/ fpp2

Hyndman R, Fan S (2010) Density forecasting for long-term peak electricity demand. IEEE Trans Power Syst 25:1142-1153

IRENA (2021) Renewable energy capacity statistics 2021. https://www.irena.org/Statistics/Downl oad-Data

Kettunen J, Salo A, Bunn DW (2010) Optimization of electricity retailer's contract portfolio subject to risk preferences. IEEE Trans Power Syst 25(1):117-128

London Datastore (2013) SmartMeter energy consumption. https://data.london.gov.uk/dataset/smart meter-energy-use-data-in-london-households

Ma P, Zhang X, Xing X, Ma J, Mahoney MW (2020) Asymptotic analysis of sampling estimators for randomized numerical linear algebra algorithms

Nojavan S, Zare K, Mohammadi-Ivatloo B (2017) Optimal stochastic energy management of retailer based on selling price determination under smart grid environment in the presence of demand response program. Appl Energy 187:449-464

OFGEM (2021) The GB electricity wholesale market. https://www.ofgem.gov.uk/electricity/wholesalemarket/gb-electricity-wholesale-market

Pineda S, Conejo A (2010) Scenario reduction for risk-averse electricity trading. Iet Gen Transm Distrib 4:694-705

Rockafellar RT, Uryasev S (2000) Optimization of conditional value-at-risk. J Risk 2:21-41

Rockafellar R, Uryasev S (2002) Conditional value-at-risk for general loss distributions. J Bank Financ 26:1443-1471

US Energy Department (2022) Demand response. https://www.energy.gov/oe/activities/technology-devel opment/grid-modernization-and-smart-grid/demand-response

Yang P, Tang G, Nehorai A (2013) A game-theoretic approach for optimal time-of-use electricity pricing. IEEE Trans Power Syst 28(2):884-892

Yang J, Zhao J, Wen F, Dong ZY (2018) A model of customizing electricity retail prices based on load profile clustering analysis. IEEE Trans Smart Grid 4:1-1 
Publisher's Note Springer Nature remains neutral with regard to jurisdictional claims in published maps and institutional affiliations.

\section{Authors and Affiliations}

\section{Román Pérez-Santalla ${ }^{1} \cdot$ Miguel Carrión $^{2} \cdot$ Carlos Ruiz $^{3}$}

\section{Carlos Ruiz}

carlos.ruiz@uc3m.es

Román Pérez-Santalla

100420252@alumnos.uc3m.es

\section{Miguel Carrión}

miguel.carrion@uclm.es

1 University Carlos III de Madrid, Avda. de la Universidad, 30, 28911 Leganés, Spain

2 Escuela de Ingeniería Industrial y Aeroespacial de Toledo, University of Castilla-La Mancha, Avda. Carlos III, 45071 Toledo, Spain

3 Department of Statistics and UC3M-BS Institute for Financial Big Data (IFiBiD), University Carlos III de Madrid, Avda. de la Universidad, 30, 28911 Leganés, Spain 